Article

\title{
Diastereoselective Three-Component Reactions of Chiral Nickel(II) Glycinate for Convenient Synthesis of Novel $\alpha$-Amino- $\beta$-Substituted- $\gamma, \gamma$-Disubstituted Butyric Acids
}

\author{
Rui Zhou ${ }^{1}$, Li Guo ${ }^{1}$, Cheng Peng ${ }^{2}$, Gu He ${ }^{1, *}$, Liang Ouyang ${ }^{1}$ and Wei Huang ${ }^{1,2, *}$
}

1 State Key Laboratory of Biotherapy, West China Hospital, and West China School of Pharmacy, Sichuan University, Chengdu 610041, Sichuan, China; E-Mails: sklb_zhourui@126.com (R.Z.); guoli@scu.edu.cn (L.G.); ouyangliang@scu.edu.cn (L.O.)

2 State Key Laboratory Breeding Base of Systematic Research, Development and Utilization of Chinese Medicine, Chengdu University of Traditional Chinese Medicine, Chengdu 610041, Sichuan, China; E-Mail: pengchengchengdu@126.com

* Authors to whom correspondence should be addressed; E-Mails: hegu@scu.edu.cn (G.H.); huangwei@cdutcm.edu.cn (W.H.); Tel./Fax: +86-28-8550-3817 (G.H.).

Received: 9 December 2013; in revised form: 20 December 2013 / Accepted: 25 December 2013 / Published: 10 January 2014

\begin{abstract}
The convenient, high yielding and diastereoselective synthesis of $\alpha$-amino- $\beta$-substituted- $\gamma, \gamma$-disubstituted butyric acid derivatives was carried out by a three-component tandem reaction of a chiral equivalent of nucleophilic glycine. The reaction was performed smoothly under mild conditions and enabled the construction of two or three adjacent chiral centers in one step, thus affording a novel and convenient route to $\alpha$-amino- $\beta$-substituted- $\gamma, \gamma$-disubstituted butyric acid derivatives.
\end{abstract}

Keywords: multi-component reaction; nickel(II); glycine; diastereoselectivity; unnatural amino acids

\section{Introduction}

Chiral $\alpha$-amino- $\gamma, \gamma$-disubstituted fragments are frequently found in various bioactive compounds, such as anti-infective agents (compound 1), anti-tuberculosis agents (compound 2), modulators of RNA binding proteins (compound 3) and compositions for specific inhibition of protein splicing by small molecules, and used in the treatment of tuberculosis and other conditions (compound 4) 
(Figure 1) [1-4]. Catalytic diastereoselective synthesis of these chiral $\alpha$-amino- $\beta$-substituted butyric acid derivatives rely on many reactions, for example, addition of $\alpha, \beta$-unsaturated acyloxazolidinones, then the removal of the oxazolidinone portions [5], cycloaddition of chiral nitrones with (E)-1,4-dichlorobut-2-ene, followed by acid-catalyzed hydrolysis and then by amide hydrolysis [6], but the Michael addition should be considered the main method to get these products when a chiral equivalent of glycine is used. Indeed, several examples of such reactions using chiral auxiliaries have been reported [7-15]. However, to our knowledge, there are no reports about the synthesis of chiral $\alpha$-amino- $\beta$-substituted- $\gamma, \gamma$-disubstituted butyric acid derivatives.

Figure 1. Structures of some biologically important compounds containing $\alpha$-amino- $\beta$ substituted $\gamma, \gamma$ - disubstituted butyric acid motifs.

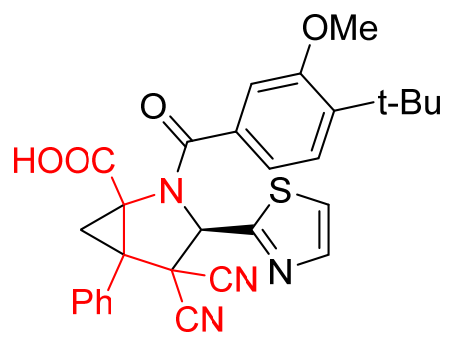

1

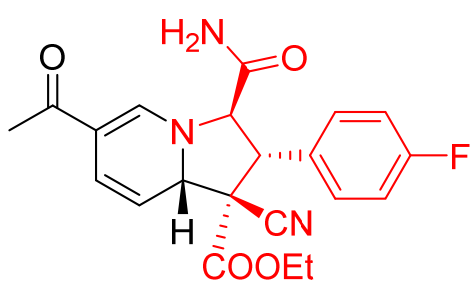

3

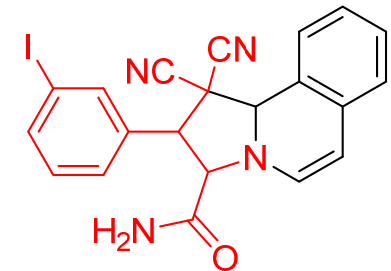

2

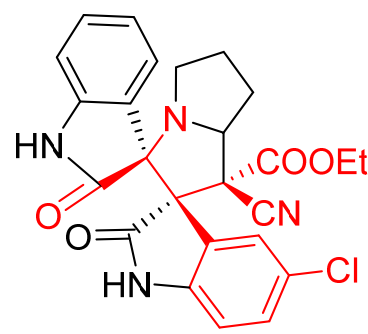

4

The chiral Ni(II) complex of the Schiff base of glycine (abbreviated as $(S)$-BPB) is commonly used in the asymmetric syntheses of unnatural amino acids. Product mixtures with a high excess of the $(S)$-amino acid diastereomer are always generated by the addition using $(S)$-BPB as a ligand [16-18]. The products can be easily isolated by column chromatography, and decomposed by acid to get chiral pure amino acids. Moreover, the recovery of $(S)$-BPB can be high (up to 85\%). To the best of our knowledge, a variety of glutamic acid and proline derivatives with a high ee values can be synthesized through Michael additions of activated olefins to Ni(II) glycinate [19-21]. Recently, Liu et al. reported the efficient synthesis of $\beta$-substituted $\alpha, \gamma$-diaminobutyric acid derivatives using asymmetric Michael addition reactions of chiral nickel(II) glycinate with nitroalkenes [22-27], Schneider et al. reported the stereoselectivity synthesis of $\gamma$-carboxyglutamic acids using asymmetric Michael addition reactions of chiral copper(II) glycinate with di-tert-butyl methylenemalonate [28]. This report focuses on the synthesis of $\alpha$-amino- $\beta$-substituted- $\gamma, \gamma$-disubstituted butyric acid derivatives through the reaction of aromatic aldehydes, a chiral $\mathrm{Ni}$ (II) glycinate complex, and an $\alpha$-carbanion of two electron-withdrawing groups (malononitrile or ethyl cyanoacetate) as a continuation of our previous research on new methods for the preparation of potentially bioactive compounds by multi-component reactions [29-33]. In the 
process, two carbon-carbon bonds were constructed and two or three chiral centers were generated in a convenient one-pot reaction with a high stereoselectivity.

\section{Results and Discussion}

The Michael addition reaction was considered as an effective way to get the products. Firstly, the optimization of the reaction conditions was undertaken using a model reaction of chiral nickel(II) glycinate with 2-benzylidenemalononitrile (Table 1). The reaction with 1,8-diazabicyclo[5.4.0]undec7-ene (DBU), triethylamine(TEA), 4-methylmorpholine (NMM) and piperidine gave a little lower diastereoselectivities (Entries 1-5, Table 1) than Hunig's base (DIEA) did, and all the reactions gave satisfactory yields, except the one in NMM.

Table 1. Optimization of the reaction conditions ${ }^{\mathrm{a}}$.

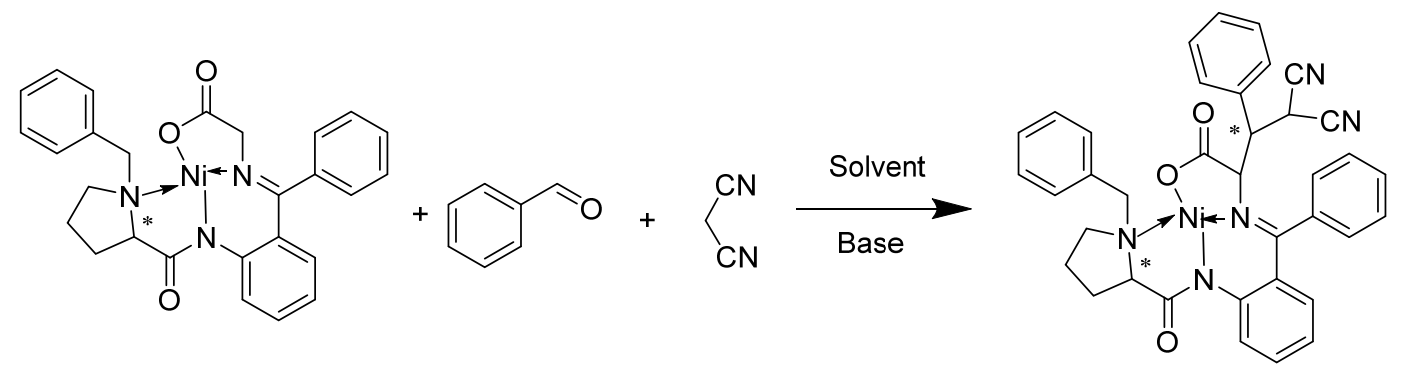

(S)-5

$6 a$

$(\mathrm{S}, 2 \mathrm{~S}, 3 \mathrm{R})-7 \mathrm{a}$

\begin{tabular}{ccccc}
\hline Entry & Base & Solvent & Yield (\%) $^{\mathbf{b}}$ & $\boldsymbol{d e}^{\mathbf{c}}$ \\
\hline 1 & DBU $^{\mathrm{d}}$ & $\mathrm{CH}_{3} \mathrm{CN}$ & 94 & $88 \%$ \\
2 & TEA & $\mathrm{CH}_{3} \mathrm{CN}$ & 97 & $94 \%$ \\
3 & DIEA & $\mathrm{CH}_{3} \mathrm{CN}$ & 94 & $96 \%$ \\
4 & $\mathrm{NMM}$ & $\mathrm{CH}_{3} \mathrm{CN}$ & 63 & $92 \%$ \\
5 & Piperidine & $\mathrm{CH}_{3} \mathrm{CN}$ & 89 & $96 \%$ \\
6 & DIEA & $\mathrm{DMF}$ & 99 & $88 \%$ \\
7 & DIEA & $\mathrm{EA}$ & 98 & $80 \%$ \\
8 & DIEA & $\mathrm{MeOH}$ & 99 & $88 \%$ \\
9 & DIEA & DCM & 97 & $58 \%$ \\
10 & DIEA & dioxane & 91 & $97 \%$ \\
11 & DIEA & $\mathrm{CHCl}$ & 59 & $90 \%$ \\
12 & DIEA & DMSO & 91 & $76 \%$ \\
\hline
\end{tabular}

${ }^{\mathrm{a}}$ All the reactions were conducted at ambient temperature; ${ }^{\mathrm{b}}$ Yield of the major products after silica gel column chromatography; ${ }^{c}$ Determined by HPLC analysis; ${ }^{\mathrm{d}}$ DBU was used in 0.15 equiv.; and other bases were used in 3 equiv.

The reaction proceeded smoothly in most of the solvents tested, although the one in chloroform gave a bad yield and the one in dichloromethane (DCM) gave poor diastereoselectivity. Good diastereoselectivities and yields were observed with the use of acetonitrile, $N, N$-dimethylformamide (DMF), ethyl acetate (EA), methanol, dioxane and dimethyl sulfoxide (DMSO). Above all, diastereoselectivity was not obviously influenced by the kind of the bases used, and polar solvents seemed to be better than nonpolar ones. As 2-benzylidenemalononitrile can be easily generated from 
benzaldehyde and malononitrile under alkaline conditions, domino reaction of these three components was thought to be feasible. In fact, TLC showed that when these three components were mixed together under basic conditions, benzaldehyde first reacted quickly with malononitrile, then added to the nickel(II) glycinate and the product 7a appeared. The results showed no big difference with those in Table 1, so the substrate scope was investigated using DIEA as the base and dioxane as the solvent (entry 11, Table 1) without further optimization.

The aromatic aldehydes with substituents at different positions were introduced into this reaction (Table 2). Whether functionalized with either electron-withdrawing or electron-donating groups, these aldehydes gave the products in good to high yields. As the result obtained with malononitrile was inspiring, ethyl cyanoacetate was introduced into the reaction, and gave a satisfactory result, so the reactions with malononitrile and ethyl cyanoacetate could be looked as two series. The results of the ethyl cyanoacetate series seemed a little better than the malononitrile series on average, despite the fact three chiral centers are newly generated. In both series, the naphthyl-functionalized aldehydes had the best diastereoselectivities (Table 2, enties 9 and 10), and ortho-functionalized aromatic aldehydes gave relatively high yields and diastereoselectivities (Table 2, entries 12, 14 and 18). The results were quite different in this two series when $t$-Bu- and 3-Cl-substituted substrates were involved (Table 2, entries 2 and 22, 3 and 15). However, furaldehyde and thienaldehyde were not tolerated (Table 2, enties 13 and 14). To elucidate the relative and absolute configurations of the products, X-ray single crystal structures of $(S, 2 S, 3 R)-7 \mathbf{a}(\mathrm{CCDC} 951535)$ and $(S, 2 S, 3 R, 4 S)-\mathbf{7 q}$ (CCDC 949234) are given below (Figure 2).

To further confirm the structure, diastereoselectivity and regioselectivity, detailed NMR spectral and X-ray analyses were carried out. The structures proposed for all products were in agreement with their NMR spectra, as discussed for compounds $\mathbf{7 a}$ and $\mathbf{7 q}$ as examples. In the ${ }^{1} \mathrm{H}-\mathrm{NMR}$ spectrum of $7 \mathbf{a}$ and $7 \mathbf{q}$, the $\alpha-\mathrm{C}$ proton of glycine exhibited double(d) peaks at $\delta 4.57(\mathrm{~d}, J=4 \mathrm{~Hz}, 1 \mathrm{H})$ and $\delta 4.60(\mathrm{~d}, J=3.6 \mathrm{~Hz}, 1 \mathrm{H})$, respectively. The $\alpha-\mathrm{C}$ proton of malononitrile in $7 \mathbf{a}$ appeared as a doublet at $\delta 5.19(\mathrm{~d}, J=12 \mathrm{~Hz}, 1 \mathrm{H})$, and the corresponding proton of ethyl cyanoacetate in $\mathbf{7 q}$ appeared as a doublet at $\delta 4.51(\mathrm{~d}, J=12.1 \mathrm{~Hz}, 1 \mathrm{H})$. The relative configuration of these structures should be as same as compound $7 \mathbf{a}$ and $\mathbf{7 q}$ shown in Figure $2 \mathrm{a}$,c, the configurations were further confirmed by the X-ray study of single crystals (Figure $2 b, d$ ). The ${ }^{13} \mathrm{C}-\mathrm{NMR}$ of compound $\mathbf{4 b}$ supported the proposed structure as well.

A plausible mechanism for the high diastereoselectivity of the reaction could be explained as follows (Scheme 1): malononitrile or cyanide ethyl acetate first reacted with aromatic aldehyde, and the intermediate formed continued to react with the complex. When $(S)$ - $N$-benzylproline was used, the benzyl group was on a certain side of this complex, so the steric hindrance was large on this side, and the intermediate would prefer attacking from the other side. Still, steric hindrance from the phenyl groups of the intermediate could contribute to the diasteroselectivity, this may explain why naphthaldehydes provided a high de value. As the diastereoselectivity was mainly controlled by the substrates, the reaction was easy to carry, making it a convenient way to get chiral $\alpha$-amino- $\beta$ substituted- $\gamma, \gamma$-disubstituted butyric acid derivatives. 
Table 2. Asymmetric Michael reactions of chiral nickel(II) glycinate ( $S$ )-5 with aromatic aldehydes and $\alpha$-carbanions ${ }^{\text {a }}$.

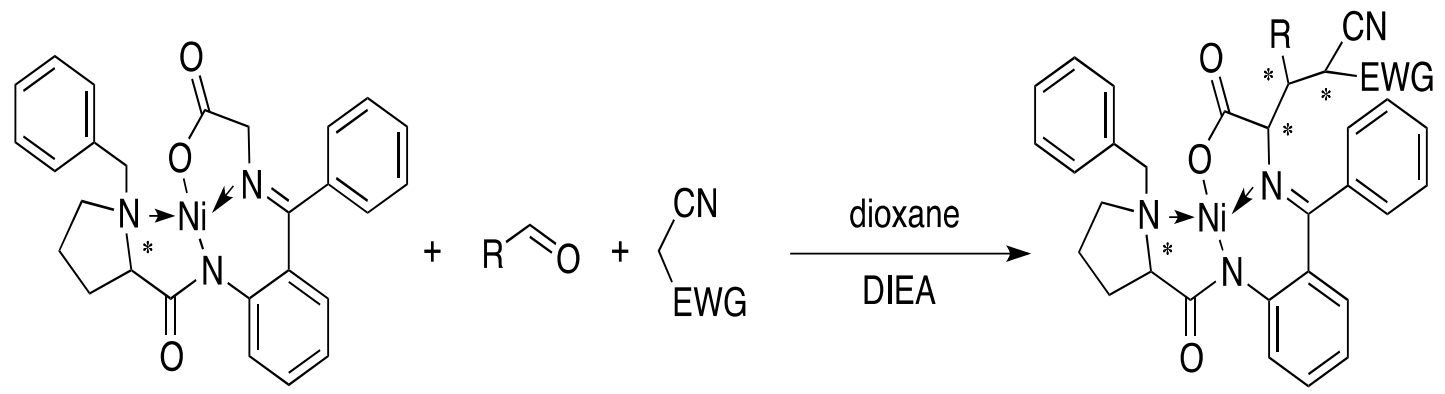

(S)-5

$(\mathrm{S}, 2 \mathrm{~S}, 3 \mathrm{R}, 4 \mathrm{~S})-7$

\begin{tabular}{|c|c|c|c|c|c|}
\hline Entry & Product & $\mathbf{R}$ & EWG & Yield (\%) ${ }^{b}$ & $d e^{\mathrm{c}}$ \\
\hline 1 & $(S, 2 S, 3 R)-7 \mathbf{a}$ & $\mathrm{Ph}$ & $\mathrm{CN}$ & 91 & $97 \%$ \\
\hline 2 & $(S, 2 S, 3 R)-7 \mathbf{b}$ & $4-(t-\mathrm{Bu})-\mathrm{C}_{6} \mathrm{H}_{4}$ & $\mathrm{CN}$ & 86 & $>99 \%$ \\
\hline 3 & $(S, 2 S, 3 R)-7 \mathbf{c}$ & $3-\mathrm{Cl}-\mathrm{C}_{6} \mathrm{H}_{4}$ & $\mathrm{CN}$ & 52 & $90 \%$ \\
\hline 4 & $(S, 2 S, 3 R)-7 \mathbf{d}$ & $4-\mathrm{F}-\mathrm{C}_{6} \mathrm{H}_{4}$ & $\mathrm{CN}$ & 83 & $93 \%$ \\
\hline 5 & $(S, 2 S, 3 R)-7 \mathbf{e}$ & 4-Br- $\mathrm{C}_{6} \mathrm{H}_{4}$ & $\mathrm{CN}$ & 44 & $98 \%$ \\
\hline 6 & $(S, 2 S, 3 R)-7 \mathbf{f}$ & $3,4-$ di-Cl- $\mathrm{C}_{6} \mathrm{H}_{3}$ & $\mathrm{CN}$ & 84 & $97 \%$ \\
\hline 7 & $(S, 2 S, 3 R)-7 \mathbf{g}$ & $3-\mathrm{Br}-\mathrm{C}_{6} \mathrm{H}_{4}$ & $\mathrm{CN}$ & 38 & $95 \%$ \\
\hline 8 & $(S, 2 S, 3 R)-7 \mathbf{h}$ & $3-\mathrm{OMe}-\mathrm{C}_{6} \mathrm{H}_{4}$ & $\mathrm{CN}$ & 82 & $>99 \%$ \\
\hline 9 & $(S, 2 S, 3 R)-7 \mathbf{i}$ & 2-naphthyl & $\mathrm{CN}$ & 80 & $98 \%$ \\
\hline 10 & $(S, 2 S, 3 R)-7 \mathbf{j}$ & 1-naphthyl & $\mathrm{CN}$ & 26 & $98 \%$ \\
\hline 11 & $(S, 2 S, 3 R)-7 \mathbf{k}$ & $3-\mathrm{OH}-\mathrm{C}_{6} \mathrm{H}_{4}$ & $\mathrm{CN}$ & 46 & $98 \%$ \\
\hline 12 & $(S, 2 S, 3 R)-7 \mathbf{l}$ & $2-\mathrm{F}-4-\mathrm{Br}-\mathrm{C}_{6} \mathrm{H}_{3}$ & $\mathrm{CN}$ & 90 & $>99 \%$ \\
\hline 13 & $(S, 2 S, 3 R, 4 S)-\mathbf{7 m}$ & $\mathrm{Ph}$ & COOEt & 78 & $98 \%$ \\
\hline 14 & $(S, 2 S, 3 R, 4 S)-\mathbf{7 n}$ & 2-Br- $\mathrm{C}_{6} \mathrm{H}_{4}$ & COOEt & 88 & $>99 \%$ \\
\hline 15 & $(S, 2 S, 3 R, 4 S)-7 \mathbf{0}$ & $3-\mathrm{Cl}-\mathrm{C}_{6} \mathrm{H}_{4}$ & COOEt & 89 & $97 \%$ \\
\hline 16 & $(S, 2 S, 3 R, 4 S)-7 \mathbf{p}$ & $4-\mathrm{F}-\mathrm{C}_{6} \mathrm{H}_{4}$ & COOEt & 75 & $96 \%$ \\
\hline 17 & $(S, 2 S, 3 R, 4 S)-\mathbf{7 q}$ & 3,4 -di-Cl- $\mathrm{C}_{6} \mathrm{H}_{3}$ & COOEt & 82 & $98 \%$ \\
\hline 18 & $(S, 2 S, 3 R, 4 S)-7 \mathbf{r}$ & 2,4-di-Cl- $\mathrm{C}_{6} \mathrm{H}_{3}$ & COOEt & 96 & $98 \%$ \\
\hline 19 & $(S, 2 S, 3 R, 4 S)-7 \mathbf{s}$ & $4-\mathrm{CH}_{3}-\mathrm{C}_{6} \mathrm{H}_{4}$ & COOEt & 76 & $>99 \%$ \\
\hline 20 & $(S, 2 S, 3 R, 4 S)-7 \mathbf{t}$ & $4-\mathrm{OCH}_{3}-\mathrm{C}_{6} \mathrm{H}_{4}$ & COOEt & 77 & $97 \%$ \\
\hline 21 & $(S, 2 S, 3 R, 4 S)-\mathbf{7 u}$ & $4-\mathrm{NO}_{2}-\mathrm{C}_{6} \mathrm{H}_{4}$ & COOEt & 69 & $97 \%$ \\
\hline 22 & $(S, 2 S, 3 R, 4 S)-\mathbf{7} \mathbf{v}$ & $4-(t-\mathrm{Bu})-\mathrm{C}_{6} \mathrm{H}_{4}$ & COOEt & 51 & $97 \%$ \\
\hline 23 & $(S, 2 S, 3 R, 4 S)-\mathbf{7} \mathbf{w}$ & 1-naphthyl & COOEt & 67 & $>99 \%$ \\
\hline 24 & $(S, 2 S, 3 R)-\mathbf{7} \mathbf{x}$ & 3-Br-thienyl & $\mathrm{CN}$ & $\mathrm{NR}^{\mathrm{d}}$ & $\mathrm{NR}^{\mathrm{d}}$ \\
\hline 25 & $(S, 2 S, 3 R)-7 \mathbf{y}$ & 4-Me-Furyl & $\mathrm{CN}$ & $\mathrm{NR}^{\mathrm{d}}$ & $\mathrm{NR}^{\mathrm{d}}$ \\
\hline
\end{tabular}

${ }^{a}$ All the reactions were conducted at ambient temperature, 3 equiv. of all the bases were used; ${ }^{b}$ Yield of the major products after silica gel column chromatography; ${ }^{\mathrm{c}}$ Determined by HPLC analysis; ${ }^{\mathrm{d}}$ Not Reaction. 
Figure 2. (a) Selected ${ }^{1} \mathrm{H}$ - and ${ }^{13} \mathrm{C}-\mathrm{NMR}$ chemical shifts of $(S, 2 S, 3 R)-7 \mathbf{a}$; (b) Single crystal X-ray diffraction study of $(S, 2 S, 3 R)-7 \mathbf{a}$; (c) Selected ${ }^{1} \mathrm{H}$ - and ${ }^{13} \mathrm{C}-\mathrm{NMR}$ chemical shifts of $(S, 2 S, 3 R, 4 S)-\mathbf{7 q}$ and (d) Single crystal X-ray diffraction study of $(S, 2 S, 3 R, 4 S)-\mathbf{7 q}$.
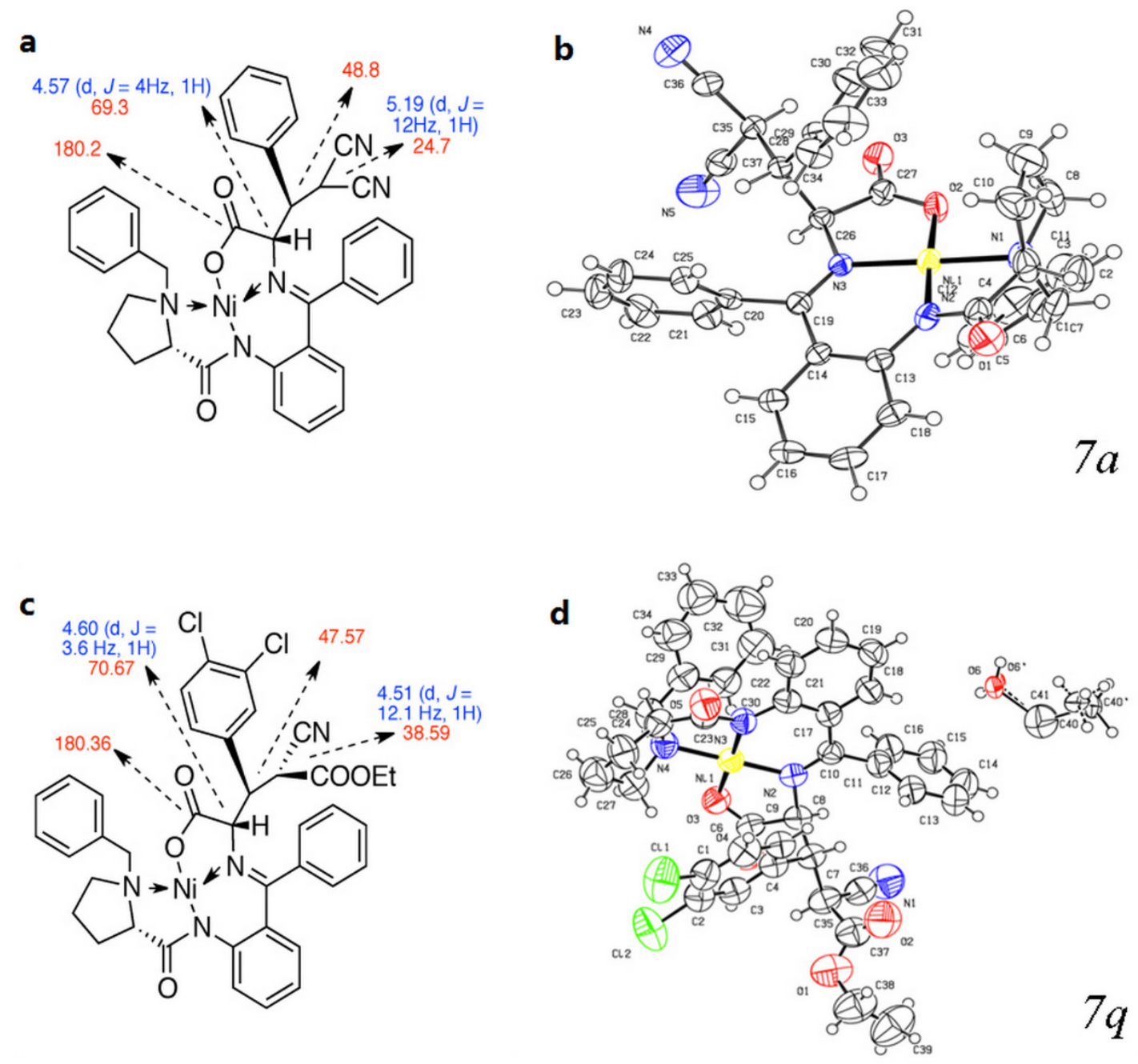

With high diastereoselectivities and mild reaction conditions, the synthesis of $(2 S, 3 R)-8 \mathbf{a}$ (Scheme 2) was completed by optimizing the metal complex decomposition and Fmoc-protection conditions. Typically, the compound $(S, 2 S, 3 R)-7 \mathbf{a}$ was decomposed by heating a suspension in methanol/6N HCl. However, we found that one of the nitrile groups was partly hydrolyzed in this process, so suitable conditions were sought to ensure that the nitrile groups remain inert. When we stirred $(S, 2 S, 3 R)-7 \mathbf{a}$ in THF with a $3 \mathrm{~N}$ concentration $\mathrm{HCl}$ at ambient temperature, the complex was decomposed and the nitrile group preserved (Scheme 2$)$. The chiral ligand $(S)$-BPB can be easily recovered quantitatively. $(2 S, 3 R)$-8a was synthesized after $(S, 2 S, 3 R)-7 \mathbf{a}$ was decomposed, the $(S)$-BPB was extracted with ethyl acetate (EA) and the $\alpha$-amino- $\beta$-substituted $\gamma, \gamma$-disubstituted butyric acid product was protected by a Fmoc group. Ultimately, the yield of $(S, 2 R)-5 \mathbf{a}$ from $(S, 2 S, 3 R)-7 \mathbf{a}$ was $62 \%$ over two steps. 
Scheme 1. Asymmetric domino reactions of chiral nickel(II) glycinate $(S)$-5 with aromatic aldehydes and $\alpha$-carbanion.

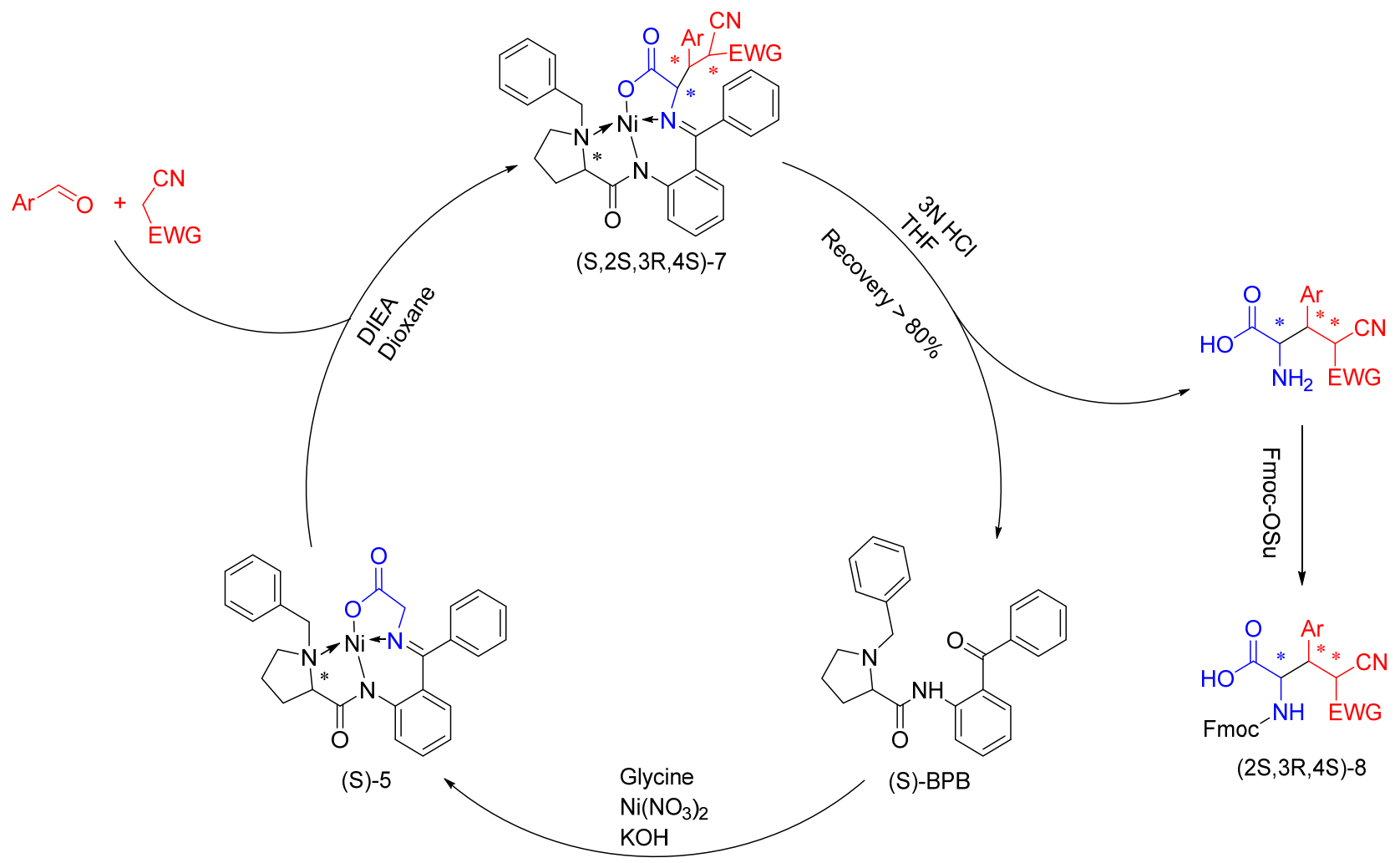

Scheme 2. Decomposition of Ni(II) complex 7a to release product 8a and recovery of the $(S)$-BPB.

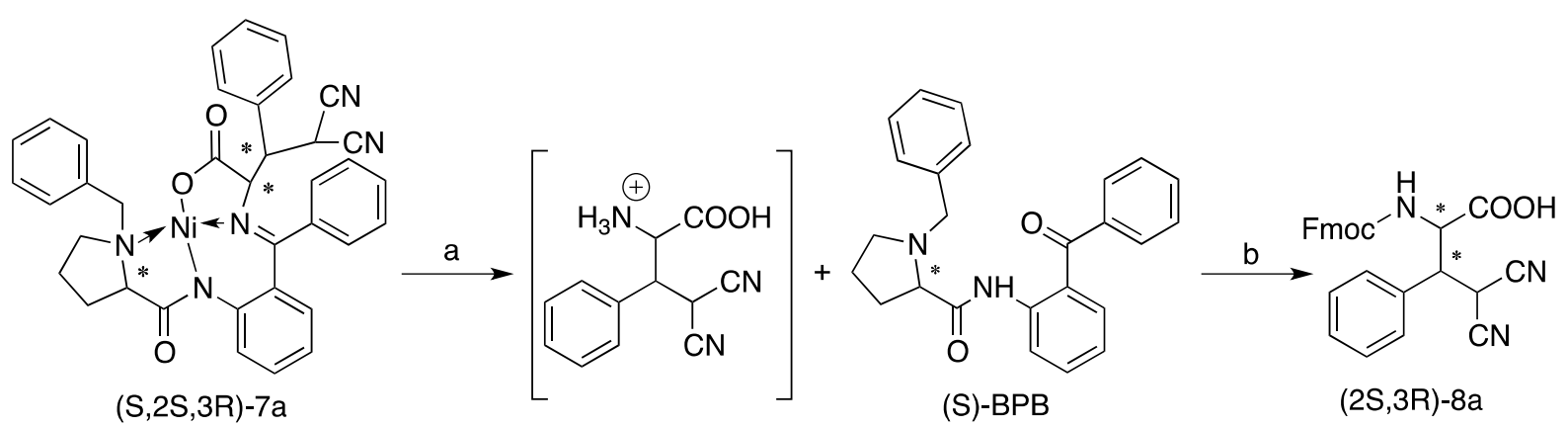

\section{Experimental}

\subsection{General}

The reagents (chemicals) were purchased from commercial sources, and used without further purification. Analytical thin layer chromatography (TLC) was $\mathrm{GF}_{254}(0.15-0.2 \mathrm{~mm}$ thickness). The mass spectra and high resolution mass spectra were obtained using Bruker microTOF-Q instrument or TOF-MS instrument. The ${ }^{1} \mathrm{H}$ - and ${ }^{13} \mathrm{C}-\mathrm{NMR}$ spectra have been respectively measured in $\mathrm{CDCl}_{3}$ or DMSO- $d_{6}$ at 400 and $100 \mathrm{MHz}$ using a Bruker Avance III $400 \mathrm{MHz}$ instrument with TMS as an internal standard. Analytical high performance liquid chromatography was carried out using the Waters Alliance 2695 HPLC, using the Chiralpak IA column. The loading loop was $10 \mu \mathrm{L}$. The eluting 
employed was an isocratic mixture of $n$-hexane and $i$-propanol (50:50 respectively) at a flow of $1 \mathrm{~mL} / \mathrm{min}$ unless stated. Retention times are reported in minutes. The enantiomeric excess was calculated from the integration of the absorption peaks at $220 \mathrm{~nm}$.

\subsection{Typical Procedure for the Synthesis of (S)-Nickel(II) Complex (5) [18]}

$(S)$-BPB (1 g, $2.60 \mathrm{mmol}), \mathrm{Ni}\left(\mathrm{NO}_{3}\right)_{2} \cdot 6 \mathrm{H}_{2} \mathrm{O}(1.52 \mathrm{~g}, 5.21 \mathrm{mmol})$ and glycine (976 mg, $\left.13.0 \mathrm{mmol}\right)$ were stirred in $\mathrm{MeOH}(50 \mathrm{~mL})$. Then $\mathrm{NaH}(55 \%-65 \%$ in oil, $1.04 \mathrm{~g}, 26 \mathrm{mmol})$ and $\mathrm{KOH}(437 \mathrm{mg}$, $7.81 \mathrm{mmol}$ ) were added successively. The mixture was refluxed for $2 \mathrm{~h}$ then cooled to room temperature and neutralized with acetic acid. After $12 \mathrm{~h}$ the precipitate was filtered and washed with ethanol $(100 \mathrm{~mL})$, followed by stirring in methane/water $(v / v)$ 1:2 $(200 \mathrm{~mL})$, then filtered to form a red crystalline solid (1.27 g, yield 98\%). The complex was sufficiently pure for further use without additional purification.

\subsection{General Procedure for the Synthesis of Ni(II) (7)}

The nickel(II) complex of glycine $(S)$-5 (1.0 equiv.) was dissolved in dioxane, and DIEA (3.0 equiv.), aromatic aldehyde (1.2 equiv.) and malononitrile/ethyl cyanoacetate (1.2 equiv.) was added at room temperature. The mixture was then stirred at room temperature for $12 \mathrm{~h}$, then poured into $10 \%$ citric acid solution, extracted with $\mathrm{CH}_{2} \mathrm{Cl}_{2}$ (three times), dried with anhydrous $\mathrm{Na}_{2} \mathrm{SO}_{4}$, concentrated, and purified on silica (petroleum ether/ethyl acetate $=1 / 1$ ) to give 7 as a red solid.

\subsubsection{Ni(II)-(S)-BPB/(2S,3R)-2-Amino-4,4'-dicyano-3-phenylbutyric Acid Schiff Base Complex (7a)}

Yield $=91 \%$, m.p. $202-204{ }^{\circ} \mathrm{C} .[\alpha]_{\mathrm{D}}^{18}=+1602\left(\right.$ ca. $\left.0.2 \mathrm{~g} / 100 \mathrm{~mL}, \mathrm{CHCl}_{3}\right) .{ }^{1} \mathrm{H}-\mathrm{NMR}\left(\mathrm{CDCl}_{3}\right) \delta 8.25$ $(\mathrm{d}, J=8 \mathrm{~Hz}, 1 \mathrm{H}), 7.98(\mathrm{~d}, J=8 \mathrm{~Hz}, 2 \mathrm{H}), 7.68-7.63(\mathrm{~m}, 6 \mathrm{H}), 7.39$ (d, $J=8 \mathrm{~Hz}, 1 \mathrm{H}), 7.30-7.26$ (m, 4H), 7.18-7.14 (m, 3H), 6.70 (d, $J=4 \mathrm{~Hz}, 1 \mathrm{H}), 5.19(\mathrm{~d}, J=12 \mathrm{~Hz}, 1 \mathrm{H}), 4.57(\mathrm{~d}, J=4 \mathrm{~Hz}, 1 \mathrm{H}), 4.15$ $(\mathrm{d}, J=12 \mathrm{~Hz}, 1 \mathrm{H}), 3.42(\mathrm{~d}, J=12 \mathrm{~Hz}, 1 \mathrm{H}), 3.29-3.21(\mathrm{~m}, 2 \mathrm{H}), 2.97-2.91(\mathrm{~m}, 1 \mathrm{H}), 2.32-2.20(\mathrm{~m}, 1 \mathrm{H})$, 2.06-2.02 (m, 1H), 1.95-1.91 (m, 1H), 1.85-1.83 (m, 1H), 1.56-1.50 (m, 1H). ${ }^{13} \mathrm{C}-\mathrm{NMR}\left(\mathrm{CDCl}_{3}\right)$ $\delta 180.2,176.2,173.5,143.4,133.9,133.5,133.2,132.6,131.4,130.9,130.6,130.1,129.9,129.5$, 128.9, 128.9, 127.5, 127.0, 125.5, 123.3, 120.8, 111.5, 111.3, 70.5, 69.7, 63.9, 57.4, 48.8, 29.7, 24.7, 23.1. ESI-MS $(m / z)$ : calcd. 652.2, found $652.2\left([\mathrm{M}+\mathrm{H}]^{+}\right)$; HRMS $(\mathrm{m} / \mathrm{z})$ : calcd. $\mathrm{C}_{37} \mathrm{H}_{32} \mathrm{~N}_{5} \mathrm{NiO}_{3}$ for 652.1859, found $652.1857\left([\mathrm{M}+\mathrm{H}]^{+}\right)$. HPLC (Chiralpak IA, $n$-hexane/i-propanol $=50 / 50$, flow rate $1.0 \mathrm{~mL} / \mathrm{min}, \lambda=220 \mathrm{~nm}), \mathrm{t}_{\text {major }}=32.3 \mathrm{~min}, \mathrm{t}_{\text {minor }}=13.4 \mathrm{~min}, d e=97 \%$.

3.3.2. Ni(II)-(S)-BPB/(2S,3R)-2-Amino-4,4'-dicyano-3-(4-tert-butyl)-phenylbutyric Acid Schiff Base Complex (7b)

Yield $=86 \%$, m.p. $197-199{ }^{\circ} \mathrm{C} .[\alpha]_{\mathrm{D}}^{18}=+1602\left(\right.$ ca. $\left.0.2 \mathrm{~g} / 100 \mathrm{~mL}, \mathrm{CHCl}_{3}\right) .{ }^{1} \mathrm{H}-\mathrm{NMR}\left(\mathrm{CDCl}_{3}\right) \delta 8.29$ $(\mathrm{d}, J=8.7 \mathrm{~Hz}, 1 \mathrm{H}), 7.95(\mathrm{~d}, J=7.5 \mathrm{~Hz}, 2 \mathrm{H}), 7.68(\mathrm{dt}, J=13.0,6.8 \mathrm{~Hz}, 3 \mathrm{H}), 7.60(\mathrm{~d}, J=8.1 \mathrm{~Hz}, 2 \mathrm{H})$, $7.41(\mathrm{~d}, J=7.2 \mathrm{~Hz}, 1 \mathrm{H}), 7.29$ (dd, $J=14.5,7.1 \mathrm{~Hz}, 3 \mathrm{H}), 7.19$ (d, $J=7.9 \mathrm{~Hz}, 3 \mathrm{H}), 7.16-7.09$ (m, 2H), $6.70(\mathrm{~d}, J=2.9 \mathrm{~Hz}, 2 \mathrm{H}), 5.16(\mathrm{~d}, J=12.0 \mathrm{~Hz}, 1 \mathrm{H}), 4.58(\mathrm{~d}, J=3.4 \mathrm{~Hz}, 1 \mathrm{H}), 4.16(\mathrm{~d}, J=12.7 \mathrm{~Hz}, 1 \mathrm{H})$, $3.79(\mathrm{~s}, 1 \mathrm{H}), 3.49(\mathrm{~d}, J=12.7 \mathrm{~Hz}, 1 \mathrm{H}), 3.28-3.13(\mathrm{~m}, 2 \mathrm{H}), 3.05-2.90(\mathrm{~m}, 1 \mathrm{H}), 2.29-2.13(\mathrm{~m}, 1 \mathrm{H})$, 2.12-2.00 (m, 1H), $1.92(\mathrm{dt}, J=27.0,8.6 \mathrm{~Hz}, 1 \mathrm{H}), 1.78(\mathrm{dt}, J=18.9,9.3 \mathrm{~Hz}, 1 \mathrm{H}), 1.54(\mathrm{~s}, 1 \mathrm{H})$, 
1.46-1.31 (m, 9H). ${ }^{13} \mathrm{C}-\mathrm{NMR}\left(\mathrm{CDCl}_{3}\right) \delta 180.2,176.3,173.3,160.9,143.2,133.9,133.4,133.3,133.2$, $131.4,130.8,130.5,129.4,128.9,128.8,127.4,127.0,125.6,124.1,123.2,120.8,115.2,111.6,111.3$, 70.6, 69.8, 64.0, 57.6, 55.4, 48.1, 30.5, 24.7, 22.9. ESI-MS $(\mathrm{m} / \mathrm{z})$ : calcd. 708.2, found $708.3\left([\mathrm{M}+\mathrm{H}]^{+}\right)$; HRMS $(m / z)$ : calcd. $\mathrm{C}_{41} \mathrm{H}_{40} \mathrm{~N}_{5} \mathrm{NiO}_{3}$ for 708.2485 , found $708.2482\left([\mathrm{M}+\mathrm{H}]^{+}\right)$. HPLC (Chiralpak IA, $n$-hexane $/ i$-propanol $=50 / 50$, flow rate $1.0 \mathrm{~mL} / \mathrm{min}, \lambda=220 \mathrm{~nm}), \mathrm{t}_{\text {major }}=30.9 \mathrm{~min}, \mathrm{t}_{\text {minor }}=17.3 \mathrm{~min}$, $d e>99 \%$.

3.3.3. Ni(II)-(S)-BPB/(2S,3R)-2-Amino-4,4'-dicyano-3-(3-chlorophenyl) Butyric Acid Schiff Base Complex (7c)

Yield $=52 \%$, m.p. $211-213{ }^{\circ} \mathrm{C} .[\alpha]_{\mathrm{D}}^{18}=+1622\left(\right.$ ca. $\left.0.2 \mathrm{~g} / 100 \mathrm{~mL}, \mathrm{CHCl}_{3}\right) .{ }^{1} \mathrm{H}-\mathrm{NMR}\left(\mathrm{CDCl}_{3}\right) \delta 8.27$ $(\mathrm{d}, J=8.7 \mathrm{~Hz}, 1 \mathrm{H}), 8.00(\mathrm{~d}, J=7.5 \mathrm{~Hz}, 2 \mathrm{H}), 7.75-7.60(\mathrm{~m}, 4 \mathrm{H}), 7.55(\mathrm{~d}, J=15.7 \mathrm{~Hz}, 1 \mathrm{H}), 7.42-7.34$ (m, 2H), $7.32(\mathrm{dd}, J=14.6,6.9 \mathrm{~Hz}, 3 \mathrm{H}), 7.22-7.09(\mathrm{~m}, 4 \mathrm{H}), 6.70(\mathrm{~d}, J=3.7 \mathrm{~Hz}, 2 \mathrm{H}), 5.14(\mathrm{~d}, J=11.9 \mathrm{~Hz}$, $1 \mathrm{H}), 4.56(\mathrm{~d}, J=3.8 \mathrm{~Hz}, 1 \mathrm{H}), 4.15(\mathrm{~d}, J=12.7 \mathrm{~Hz}, 1 \mathrm{H}), 3.43$ (d, $J=12.6 \mathrm{~Hz}, 1 \mathrm{H}), 3.25$ (ddd, $J=16.2$, $10.7,5.6 \mathrm{~Hz}, 2 \mathrm{H}), 3.02(\mathrm{dd}, J=9.8,5.5 \mathrm{~Hz}, 1 \mathrm{H}), 2.25(\mathrm{dd}, J=18.6,10.0 \mathrm{~Hz}, 1 \mathrm{H}), 2.10-1.98(\mathrm{~m}, 3 \mathrm{H})$, $1.70-1.63(\mathrm{~m}, 1 \mathrm{H}) .{ }^{13} \mathrm{C}-\mathrm{NMR}\left(\mathrm{CDCl}_{3}\right) \delta 180.2,175.9,173.7,143.4,136.3,134.6,133.9,133.4,133.4$, 133.2, 131.3, 131.0, 131.0, 130.6, 130.4, 129.5, 129.0, 128.8, 127.4, 127.0, 125.4, 123.3, 120.8, 111.2, $110.9,70.5,69.4,64.0,57.6,48.4,30.7,29.6,24.6,23.0$. ESI-MS $(\mathrm{m} / \mathrm{z})$ : calcd. 686.1 , found 686.2 $\left([\mathrm{M}+\mathrm{H}]^{+}\right)$. $\quad$ HRMS $(\mathrm{m} / z)$ : calcd. $\mathrm{C}_{37} \mathrm{H}_{31} \mathrm{ClN}_{5} \mathrm{NiO}_{3}$ for 686.1469 , found $686.1475\left([\mathrm{M}+\mathrm{H}]^{+}\right)$. HPLC (Chiralpak IA, $n$-hexane $/ i$-propanol $=50 / 50$, flow rate $1.0 \mathrm{~mL} / \mathrm{min}, \lambda=220 \mathrm{~nm}$ ), $\mathrm{t}_{\text {major }}=31.0 \mathrm{~min}$, $\mathrm{t}_{\text {minor }}=13.0 \mathrm{~min}, \mathrm{de}=90 \%$.

3.3.4. Ni(II)-(S)-BPB/(2S,3R)-2-Amino-4,4'-dicyano-3-(4-fluorophenyl)butyric Acid Schiff Base Complex (7d)

Yield $=83 \%$, m.p. $207-209^{\circ} \mathrm{C} .[\alpha]_{\mathrm{D}}^{18}=+1734\left(\right.$ ca. $\left.0.2 \mathrm{~g} / 100 \mathrm{~mL}, \mathrm{CHCl}_{3}\right) .{ }^{1} \mathrm{H}-\mathrm{NMR}\left(\mathrm{CDCl}_{3}\right) \delta 8.25$ $(\mathrm{d}, J=8.6 \mathrm{~Hz}, 1 \mathrm{H}), 8.00(\mathrm{~d}, J=7.4 \mathrm{~Hz}, 2 \mathrm{H}), 7.73-7.62(\mathrm{~m}, 3 \mathrm{H}), 7.34(\mathrm{ddd}, J=14.8,13.7,7.2 \mathrm{~Hz}, 7 \mathrm{H})$, $7.21-7.09(\mathrm{~m}, 3 \mathrm{H}), 6.73-6.66(\mathrm{~m}, 2 \mathrm{H}), 5.16(\mathrm{~d}, J=12.0 \mathrm{~Hz}, 1 \mathrm{H}), 4.55(\mathrm{~d}, J=3.7 \mathrm{~Hz}, 1 \mathrm{H}), 4.15(\mathrm{~d}$, $J=13.1 \mathrm{~Hz}, 1 \mathrm{H}), 3.48-3.37(\mathrm{~m}, 1 \mathrm{H}), 3.32-3.22(\mathrm{~m}, 2 \mathrm{H}), 3.02-2.90(\mathrm{~m}, 1 \mathrm{H}), 2.33-2.23(\mathrm{~m}, 1 \mathrm{H})$, 2.13-2.05 (m, 1H), $1.97(\mathrm{dd}, J=13.3,7.4 \mathrm{~Hz}, 2 \mathrm{H}), 1.67-1.61(\mathrm{~m}, 1 \mathrm{H}) .{ }^{13} \mathrm{C}-\mathrm{NMR}\left(\mathrm{CDCl}_{3}\right) \delta 180.2$, 176.1, 173.6, 165.1, 162.6, 143.3, 133.9, 133.4, 133.3, 133.2, 131.3, 131.2, 130.9, 130.6, 129.5, 129.0, 128.8, 128.4, 128.4, 127.3, 127.0, 125.4, 123.3, 120.8, 117.1, 116.9, 111.3, 111.0, 70.5, 69.6, 64.0, 57.5, 48.1, 30.6, 24.7, 22.9. ESI-MS $(\mathrm{m} / \mathrm{z})$ : calcd. 670.2, found $670.2\left([\mathrm{M}+\mathrm{H}]^{+}\right)$. HRMS $(\mathrm{m} / \mathrm{z})$ : calcd. $\mathrm{C}_{37} \mathrm{H}_{31} \mathrm{FN}_{5} \mathrm{NiO}_{3}$ for 670.1764 , found $670.1801\left([\mathrm{M}+\mathrm{H}]^{+}\right)$. HPLC (Chiralpak IA, $n$-hexane/ $i$-propanol $=50 / 50$, flow rate $1.0 \mathrm{~mL} / \mathrm{min}, \lambda=220 \mathrm{~nm}), \mathrm{t}_{\text {major }}=26.0 \mathrm{~min}, \mathrm{t}_{\text {minor }}=10.1 \mathrm{~min}, d e=93 \%$.

3.3.5. Ni(II)-(S)-BPB/(2S,3R)-2-amino-4,4'-dicyano-3-(4-bromophenyl) Butyric Acid Schiff Base Complex (7e)

Yield $=44 \%$, m.p. ${ }^{197-199}{ }^{\circ} \mathrm{C} .[\alpha]_{\mathrm{D}}^{18}=+1972\left(\right.$ ca. $\left.0.2 \mathrm{~g} / 100 \mathrm{~mL}, \mathrm{CHCl}_{3}\right) .{ }^{1} \mathrm{H}-\mathrm{NMR}\left(\mathrm{CDCl}_{3}\right) \delta 8.23$ $(\mathrm{d}, J=8.7 \mathrm{~Hz}, 1 \mathrm{H}), 8.01(\mathrm{~d}, J=7.4 \mathrm{~Hz}, 2 \mathrm{H}), 7.78-7.58(\mathrm{~m}, 3 \mathrm{H}), 7.38(\mathrm{~d}, J=6.8 \mathrm{~Hz}, 1 \mathrm{H}), 7.29(\mathrm{dd}$, $J=14.9,7.4 \mathrm{~Hz}, 3 \mathrm{H}), 7.25-7.08(\mathrm{~m}, 7 \mathrm{H}), 6.70(\mathrm{~d}, J=13.7 \mathrm{~Hz}, 2 \mathrm{H}), 5.14(\mathrm{~d}, J=12.0 \mathrm{~Hz}, 1 \mathrm{H}), 4.53(\mathrm{~d}$, $J=3.2 \mathrm{~Hz}, 1 \mathrm{H}), 4.15(\mathrm{~d}, J=12.7 \mathrm{~Hz}, 1 \mathrm{H}), 3.40$ (d, $J=12.6 \mathrm{~Hz}, 1 \mathrm{H}), 3.31-3.18(\mathrm{~m}, 2 \mathrm{H}), 2.99$ (dd, 
$J=10.5,6.0 \mathrm{~Hz}, 1 \mathrm{H}), 2.23(\mathrm{dt}, J=24.1,12.1 \mathrm{~Hz}, 1 \mathrm{H}), 2.13-2.02(\mathrm{~m}, 1 \mathrm{H}), 2.02-1.87(\mathrm{~m}, 2 \mathrm{H}), 1.55$ $(\mathrm{dd}, J=12.1,6.5 \mathrm{~Hz}, 1 \mathrm{H}) .{ }^{13} \mathrm{C}-\mathrm{NMR}\left(\mathrm{CDCl}_{3}\right) \delta 180.2,176.3,173.3,160.9,143.2,133.9,133.4,133.3$, 133.2, 131.4, 130.8, 130.5, 129.4, 128.9, 128.8, 127.4, 127.0, 125.6, 124.1, 123.2, 120.8, 115.2, 111.6, 111.3, 70.6, 69.8, 64.0, 57.6, 55.4, 48.1, 30.5, 24.7, 22.9. ESI-MS $(\mathrm{m} / \mathrm{z})$ : calcd. 730.1, found 730.2 $\left([\mathrm{M}+\mathrm{H}]^{+}\right)$. HRMS $(m / z)$ : calcd. $\mathrm{C}_{37} \mathrm{H}_{31} \mathrm{BrN}_{5} \mathrm{NiO}_{3}$ for 730.0966 , found $730.0978\left([\mathrm{M}+\mathrm{H}]^{+}\right)$. HPLC (Chiralpak IA, $n$-hexane $/ i$-propanol $=50 / 50$, flow rate $1.0 \mathrm{~mL} / \mathrm{min}, \lambda=220 \mathrm{~nm}$ ), $\mathrm{t}_{\text {major }}=32.0 \mathrm{~min}$, $\mathrm{t}_{\text {minor }}=15.3 \mathrm{~min}, d e=98 \%$.

3.3.6. Ni(II)-(S)-BPB/(2S,3R)-2-Amino-4,4'-dicyano-3-(3,4-dichlorophenyl) Butyric Acid Schiff Base Complex (7f)

Yield $=84 \%$, m.p. $213-215^{\circ} \mathrm{C} .[\alpha]_{\mathrm{D}}^{18}=+1660\left(\right.$ ca. $\left.0.2 \mathrm{~g} / 100 \mathrm{~mL}, \mathrm{CHCl}_{3}\right) .{ }^{1} \mathrm{H}-\mathrm{NMR}\left(\mathrm{CDCl}_{3}\right) \delta 8.27$ $(\mathrm{d}, J=8.7 \mathrm{~Hz}, 1 \mathrm{H}), 8.01(\mathrm{~d}, J=7.4 \mathrm{~Hz}, 2 \mathrm{H}), 7.69(\mathrm{dd}, J=17.4,7.8 \mathrm{~Hz}, 4 \mathrm{H}), 7.44(\mathrm{~s}, 1 \mathrm{H}), 7.38(\mathrm{~d}$, $J=6.3 \mathrm{~Hz}, 1 \mathrm{H}), 7.31$ (t, $J=7.4 \mathrm{~Hz}, 2 \mathrm{H}), 7.18(\mathrm{dd}, J=15.9,8.3 \mathrm{~Hz}, 2 \mathrm{H}), 7.09$ (t, $J=7.8 \mathrm{~Hz}, 2 \mathrm{H}), 6.71$ $(\mathrm{d}, J=14.3 \mathrm{~Hz}, 2 \mathrm{H}), 5.15(\mathrm{~d}, J=11.9 \mathrm{~Hz}, 1 \mathrm{H}), 4.53(\mathrm{~s}, 1 \mathrm{H}), 4.12(\mathrm{dd}, J=17.0,9.9 \mathrm{~Hz}, 1 \mathrm{H}), 3.42(\mathrm{~d}$, $J=12.6 \mathrm{~Hz}, 1 \mathrm{H}), 3.30(\mathrm{t}, J=8.3 \mathrm{~Hz}, 1 \mathrm{H}), 3.22(\mathrm{~d}, J=11.9 \mathrm{~Hz}, 1 \mathrm{H}), 2.99(\mathrm{~d}, J=5.8 \mathrm{~Hz}, 1 \mathrm{H}), 2.33(\mathrm{dd}$, $J=20.4,8.8 \mathrm{~Hz}, 1 \mathrm{H}), 2.02(\mathrm{qd}, J=13.8,6.8 \mathrm{~Hz}, 3 \mathrm{H}), 1.77-1.66(\mathrm{~m}, 1 \mathrm{H}) .{ }^{13} \mathrm{C}-\mathrm{NMR}\left(\mathrm{CDCl}_{3}\right) \delta 180.2$, $175.8,173.8,143.4,135.0,134.6,134.0,133.5,133.3,132.7,131.7,131.3,131.1,130.7,129.6,129.0$, 128.9, 127.2, 126.9, 125.2, 123.3, 120.8, 111.1, 110.6, 70.5, 69.3, 64.1, 57.8, 48.0, 30.7, 24.4, 22.8. ESI-MS $(m / z)$ : calcd. 720.1 , found $720.2\left([\mathrm{M}+\mathrm{H}]^{+}\right)$; HRMS $(\mathrm{m} / z)$ : calcd. $\mathrm{C}_{37} \mathrm{H}_{30} \mathrm{Cl}_{2} \mathrm{~N}_{5} \mathrm{NiO}_{3}$ for 720.1079 , found $720.1090\left([\mathrm{M}+\mathrm{H}]^{+}\right)$. HPLC (Chiralpak IA, $n$-hexane/i-propanol $=50 / 50$, flow rate $1.0 \mathrm{~mL} / \mathrm{min}, \lambda=220 \mathrm{~nm}), \mathrm{t}_{\text {major }}=26.0 \mathrm{~min}, \mathrm{t}_{\text {minor }}=12.5 \mathrm{~min}, d e=97 \%$.

3.3.7. Ni(II)-(S)-BPB/(2S,3R)-2-Amino-4,4'-dicyano-3-(3-bromophenyl) Butyric Acid Schiff Base Complex (7g)

Yield $=38 \%$, m.p. $208-210{ }^{\circ} \mathrm{C} .[\alpha]_{\mathrm{D}}^{18}=+1882\left(\right.$ ca. $\left.0.2 \mathrm{~g} / 100 \mathrm{~mL}, \mathrm{CHCl}_{3}\right) .{ }^{1} \mathrm{H}-\mathrm{NMR}\left(\mathrm{CDCl}_{3}\right) \delta 8.28$ $(\mathrm{d}, J=8.7 \mathrm{~Hz}, 1 \mathrm{H}), 8.00(\mathrm{~d}, J=7.4 \mathrm{~Hz}, 2 \mathrm{H}), 7.80(\mathrm{~d}, J=8.0 \mathrm{~Hz}, 1 \mathrm{H}), 7.75-7.60(\mathrm{~m}, 3 \mathrm{H}), 7.49$ (t, $J=7.8 \mathrm{~Hz}, 2 \mathrm{H}), 7.39(\mathrm{~d}, J=6.7 \mathrm{~Hz}, 1 \mathrm{H}), 7.31(\mathrm{t}, J=7.6 \mathrm{~Hz}, 2 \mathrm{H}), 7.24-7.08(\mathrm{~m}, 4 \mathrm{H}), 6.70(\mathrm{~d}$, $J=4.0 \mathrm{~Hz}, 2 \mathrm{H}), 5.14(\mathrm{~d}, J=11.9 \mathrm{~Hz}, 1 \mathrm{H}), 4.55(\mathrm{~d}, J=3.7 \mathrm{~Hz}, 1 \mathrm{H}), 4.20-4.09(\mathrm{~m}, 1 \mathrm{H}), 3.42(\mathrm{~d}$, $J=12.6 \mathrm{~Hz}, 1 \mathrm{H}), 3.25(\mathrm{ddd}, J=15.7,10.7,5.6 \mathrm{~Hz}, 2 \mathrm{H}), 3.03(\mathrm{dd}, J=10.0,5.6 \mathrm{~Hz}, 1 \mathrm{H}), 2.25$ (dt, $J=16.4,8.4 \mathrm{~Hz}, 1 \mathrm{H}), 2.15-1.91(\mathrm{~m}, 4 \mathrm{H}) .{ }^{13} \mathrm{C}-\mathrm{NMR}\left(\mathrm{CDCl}_{3}\right) \delta 180.2,176.3,173.3,160.9,143.2,133.9$, 133.4, 133.3, 133.2, 131.4, 130.8, 130.5, 129.4, 128.9, 128.8, 127.4, 127.0, 125.6, 124.1, 123.2, 120.8, 115.2, 111.6, 111.3, 70.6, 69.8, 64.0, 57.6, 55.4, 48.1, 30.5, 24.7, 22.9. ESI-MS ( $\mathrm{m} / \mathrm{z})$ : calcd. 730.1, found $730.2\left([\mathrm{M}+\mathrm{H}]^{+}\right)$; HRMS $(\mathrm{m} / z)$ : calcd. $\mathrm{C}_{37} \mathrm{H}_{31} \mathrm{BrN}_{5} \mathrm{NiO}_{3}$ for 730.0966 , found $730.0966\left([\mathrm{M}+\mathrm{H}]^{+}\right)$. HPLC (Chiralpak IA, $n$-hexane $/ i$-propanol $=50 / 50$, flow rate $1.0 \mathrm{~mL} / \mathrm{min}, \lambda=220 \mathrm{~nm}$ ), $\mathrm{t}_{\text {major }}=32.0$, $\mathrm{t}_{\text {minor }}=14.0 \mathrm{~min}, \mathrm{de}=95 \%$.

3.3.8. Ni(II)-(S)-BPB/(2S,3R)-2-Amino-4,4'-dicyano-3-(3-methoxyphenyl) Butyric Acid Schiff Base Complex (7h)

Yield $=82 \%$, m.p. $222-224{ }^{\circ} \mathrm{C} .[\alpha]_{\mathrm{D}}^{18}=+1678\left(\right.$ ca. $\left.0.2 \mathrm{~g} / 100 \mathrm{~mL}, \mathrm{CHCl}_{3}\right) .{ }^{1} \mathrm{H}-\mathrm{NMR}\left(\mathrm{CDCl}_{3}\right) \delta 8.18$ $(\mathrm{d}, J=8.7 \mathrm{~Hz}, 1 \mathrm{H}), 7.93(\mathrm{~d}, J=7.5 \mathrm{~Hz}, 2 \mathrm{H}), 7.59(\mathrm{dd}, J=13.8,7.2 \mathrm{~Hz}, 3 \mathrm{H}), 7.44(\mathrm{t}, J=7.9 \mathrm{~Hz}, 1 \mathrm{H})$, 
$7.32(\mathrm{~d}, J=6.9 \mathrm{~Hz}, 1 \mathrm{H}), 7.23(\mathrm{t}, J=7.5 \mathrm{~Hz}, 2 \mathrm{H}), 7.07(\mathrm{dd}, J=13.9,6.8 \mathrm{~Hz}, 4 \mathrm{H}), 6.75(\mathrm{~d}, J=11.2 \mathrm{~Hz}$, 2H), $6.62(\mathrm{~d}, J=4.0 \mathrm{~Hz}, 2 \mathrm{H}), 5.09(\mathrm{~d}, J=12.0 \mathrm{~Hz}, 1 \mathrm{H}), 4.47(\mathrm{~d}, J=3.4 \mathrm{~Hz}, 1 \mathrm{H}), 4.04(\mathrm{dd}, J=15.5$, $9.7 \mathrm{~Hz}, 1 \mathrm{H}), 3.71(\mathrm{~s}, 3 \mathrm{H}), 3.34(\mathrm{~d}, J=12.6 \mathrm{~Hz}, 1 \mathrm{H}), 3.16(\mathrm{dd}, J=16.1,6.4 \mathrm{~Hz}, 2 \mathrm{H}), 2.89$ (q, $J=10.2 \mathrm{~Hz}$, $1 \mathrm{H}), 2.26-2.06(\mathrm{~m}, 1 \mathrm{H}), 2.01(\mathrm{dd}, J=13.8,7.1 \mathrm{~Hz}, 1 \mathrm{H}), 1.87(\mathrm{dd}, J=18.6,6.4 \mathrm{~Hz}, 2 \mathrm{H}), 1.49$ (dd, $J=14.2,9.1 \mathrm{~Hz}, 1 \mathrm{H}) .{ }^{13} \mathrm{C}-\mathrm{NMR}\left(\mathrm{CDCl}_{3}\right) \delta 180.1,176.2,173.3,160.6,143.3,133.9,133.8,133.4$, $133.3,133.2,131.4,130.9,130.9,130.5,129.4,128.9,128.8,127.5,127.0,125.5,123.2,120.7,115.7$, $111.5,111.2,70.5,69.6,63.9,57.4,55.3,48.6,30.6,24.6,23.0$. ESI-MS $(\mathrm{m} / \mathrm{z})$ : calcd. 682.2 , found $682.3\left([\mathrm{M}+\mathrm{H}]^{+}\right)$; HRMS $(\mathrm{m} / \mathrm{z})$ : calcd. $\mathrm{C}_{38} \mathrm{H}_{34} \mathrm{~N}_{5} \mathrm{NiO}_{4}$ for 682.1964, found $682.1959\left([\mathrm{M}+\mathrm{H}]^{+}\right)$. HPLC (Chiralpak IA, $n$-hexane $/ i$-propanol $=50 / 50$, flow rate $1.0 \mathrm{~mL} / \mathrm{min}, \lambda=220 \mathrm{~nm}$ ), $\mathrm{t}_{\text {major }}=36.2$, $\mathrm{t}_{\text {minor }}=16.5 \mathrm{~min}, \mathrm{de}>99 \%$.

3.3.9. Ni(II)-(S)-BPB/(2S,3R)-2-Amino-4,4'-dicyano-3-(2-naphthyl) Butyric Acid Schiff Base Complex (7i)

Obtained as a red solid, yield $=80 \%$, m.p. $187-189{ }^{\circ} \mathrm{C} .[\alpha]_{\mathrm{D}}^{18}=+1582$ (ca. $0.2 \mathrm{~g} / 100 \mathrm{~mL}, \mathrm{CHCl}_{3}$ ). ${ }^{1} \mathrm{H}-\mathrm{NMR}\left(\mathrm{CDCl}_{3}\right) \delta 8.19(\mathrm{~d}, J=8.7 \mathrm{~Hz}, 1 \mathrm{H}), 8.08(\mathrm{~d}, J=8.4 \mathrm{~Hz}, 1 \mathrm{H}), 7.97(\mathrm{~d}, J=7.5 \mathrm{~Hz}, 3 \mathrm{H}), 7.93$ $(\mathrm{d}, J=7.7 \mathrm{~Hz}, 1 \mathrm{H}), 7.81(\mathrm{~s}, 1 \mathrm{H}), 7.70(\mathrm{~d}, J=7.8 \mathrm{~Hz}, 3 \mathrm{H}), 7.60(\mathrm{p}, J=6.7 \mathrm{~Hz}, 2 \mathrm{H}), 7.41(\mathrm{~d}$, $J=7.0 \mathrm{~Hz}, 1 \mathrm{H}), 7.32(\mathrm{~d}, J=7.0 \mathrm{~Hz}, 1 \mathrm{H}), 7.26(\mathrm{t}, J=7.6 \mathrm{~Hz}, 3 \mathrm{H}), 7.20-7.09(\mathrm{~m}, 2 \mathrm{H}), 6.70(\mathrm{~d}$, $J=14.1 \mathrm{~Hz}, 2 \mathrm{H}), 5.34(\mathrm{~d}, J=12.0 \mathrm{~Hz}, 1 \mathrm{H}), 4.62(\mathrm{~d}, J=3.1 \mathrm{~Hz}, 1 \mathrm{H}), 4.12(\mathrm{~d}, J=7.1 \mathrm{~Hz}, 1 \mathrm{H}), 4.02(\mathrm{~d}$, $J=12.5 \mathrm{~Hz}, 1 \mathrm{H}), 3.46(\mathrm{dd}, J=12.0,3.1 \mathrm{~Hz}, 1 \mathrm{H}), 3.23(\mathrm{~d}, J=12.5 \mathrm{~Hz}, 1 \mathrm{H}), 3.05(\mathrm{t}, J=8.5 \mathrm{~Hz}, 1 \mathrm{H})$, 2.64-2.48 (m, 1H), $2.04(\mathrm{~s}, 1 \mathrm{H}), 1.74(\mathrm{~d}, J=10.0 \mathrm{~Hz}, 2 \mathrm{H}), 1.24(\mathrm{dd}, J=16.0,8.8 \mathrm{~Hz}, 1 \mathrm{H}) .{ }^{13} \mathrm{C}-\mathrm{NMR}$ $\left(\mathrm{CDCl}_{3}\right) \delta 180.1,176.2,173.4,143.3,134.1,133.9,133.5,133.3,133.2,131.3,130.9,130.5,129.8$, $129.5,128.9,128.8,128.5,127.8,127.5,127.5,127.3,127.1,125.5,123.3,120.7,111.6,111.3,70.3$, 70.0, 64.0, 57.7, 48.9, 30.0, 24.6, 22.3. ESI-MS $(\mathrm{m} / \mathrm{z})$ : calcd. 702.2, found $702.3\left([\mathrm{M}+\mathrm{H}]^{+}\right)$; HRMS $(m / z)$ : calcd. $\mathrm{C}_{41} \mathrm{H}_{34} \mathrm{~N}_{5} \mathrm{NiO}_{3}$ for 702.2015 , found $702.2022\left([\mathrm{M}+\mathrm{H}]^{+}\right)$. HPLC (Chiralpak IA, $n$-hexane/ $i$-propanol $=50 / 50$, flow rate $1.0 \mathrm{~mL} / \mathrm{min}, \lambda=220 \mathrm{~nm}), \mathrm{t}_{\text {major }}=31.5 \mathrm{~min}, \mathrm{t}_{\text {minor }}=18.8 \mathrm{~min}, \mathrm{de}=98 \%$.

3.3.10. Ni(II)-(S)-BPB/(2S,3R)-2-Amino-4,4'-dicyano-3-(1-naphthyl) Butyric Acid Schiff Base Complex (7j)

Yield $=26 \%$, m.p. $178-180{ }^{\circ} \mathrm{C} .[\alpha]_{\mathrm{D}}^{18}=+1614\left(\right.$ ca. $\left.0.2 \mathrm{~g} / 100 \mathrm{~mL}, \mathrm{CHCl}_{3}\right) .{ }^{1} \mathrm{H}-\mathrm{NMR}\left(\mathrm{CDCl}_{3}\right) \delta 8.25$ $(\mathrm{d}, J=8.8 \mathrm{~Hz}, 1 \mathrm{H}), 8.14(\mathrm{~d}, J=8.2 \mathrm{~Hz}, 1 \mathrm{H}), 8.05(\mathrm{~d}, J=8.2 \mathrm{~Hz}, 1 \mathrm{H}), 7.89(\mathrm{~d}, J=7.5 \mathrm{~Hz}, 2 \mathrm{H})$, $7.80-7.65(\mathrm{~m}, 4 \mathrm{H}), 7.57(\mathrm{~d}, J=7.3 \mathrm{~Hz}, 1 \mathrm{H}), 7.55-7.43(\mathrm{~m}, 3 \mathrm{H}), 7.41(\mathrm{~d}, J=7.0 \mathrm{~Hz}, 1 \mathrm{H}), 7.29-7.21$ (m, 3H), 7.21-7.07 (m, 3H), 6.80-6.67 (m, 2H), $5.36(\mathrm{~d}, J=11.8 \mathrm{~Hz}, 1 \mathrm{H}), 4.74(\mathrm{~d}, J=2.6 \mathrm{~Hz}, 1 \mathrm{H})$, $4.20(\mathrm{dd}, J=11.8,2.2 \mathrm{~Hz}, 1 \mathrm{H}), 4.12(\mathrm{q}, J=7.1 \mathrm{~Hz}, 1 \mathrm{H}), 3.97(\mathrm{~d}, J=12.6 \mathrm{~Hz}, 1 \mathrm{H}), 3.25(\mathrm{~d}, J=12.6 \mathrm{~Hz}$, $1 \mathrm{H}), 2.95(\mathrm{t}, J=8.7 \mathrm{~Hz}, 1 \mathrm{H}), 2.50(\mathrm{dt}, J=11.4,5.8 \mathrm{~Hz}, 1 \mathrm{H}), 1.86(\mathrm{dd}, J=12.9,9.2 \mathrm{~Hz}, 1 \mathrm{H}), 1.75$ (dt, $J=22.7,9.6 \mathrm{~Hz}, 2 \mathrm{H}), 0.97(\mathrm{dt}, J=14.6,7.4 \mathrm{~Hz}, 1 \mathrm{H}) .{ }^{13} \mathrm{C}-\mathrm{NMR}\left(\mathrm{CDCl}_{3}\right) \delta 179.5,176.1,173.6,143.4$, $134.4,134.0,133.5,133.4,133.1,133.0,131.2,130.6,130.4,130.1,129.3,129.2,128.8,128.7,127.2$, 127.1, 126.9, 126.7, 126.1, 126.0, 125.1, 123.0, 122.5, 120.5, 111.6, 111.1, 71.4, 70.3, 63.7, 57.2, 43.5, 30.2, 25.7, 22.9. ESI-MS $(\mathrm{m} / \mathrm{z})$ : calcd. 702.2, found $702.3 \quad\left([\mathrm{M}+\mathrm{H}]^{+}\right)$; HRMS $(\mathrm{m} / \mathrm{z})$ : calcd. $\mathrm{C}_{41} \mathrm{H}_{34} \mathrm{~N}_{5} \mathrm{NiO}_{3}$ for 702.2015 , found $702.2019\left([\mathrm{M}+\mathrm{H}]^{+}\right)$. HPLC (Chiralpak IA, $n$-hexane/ $i$-propanol $=50 / 50$, flow rate $1.0 \mathrm{~mL} / \mathrm{min}, \lambda=220 \mathrm{~nm}), \mathrm{t}_{\text {major }}=55.7 \mathrm{~min}, \mathrm{t}_{\text {minor }}=20.4 \mathrm{~min}, d e=98 \%$. 
3.3.11. Ni(II)-(S)-BPB/(2S,3R)-2-Amino-4,4'-dicyano-3-(3-hydroxyphenyl) Butyric Acid Schiff Base Complex (7k)

Yield $=46 \%$, m.p. $222-224{ }^{\circ} \mathrm{C} .[\alpha]_{\mathrm{D}}^{18}=+1775$ (ca. $0.2 \mathrm{~g} / 100 \mathrm{~mL}, \mathrm{CHCl}_{3}$ ). ${ }^{1} \mathrm{H}-\mathrm{NMR}$ (DMSO) $\delta 9.89$ $(\mathrm{s}, 1 \mathrm{H}), 8.42(\mathrm{~d}, J=7.5 \mathrm{~Hz}, 2 \mathrm{H}), 8.11(\mathrm{~d}, J=8.7 \mathrm{~Hz}, 1 \mathrm{H}), 7.78(\mathrm{~d}, J=5.3 \mathrm{~Hz}, 1 \mathrm{H}), 7.69$ (s, 3H), 7.54 $(\mathrm{d}, J=4.9 \mathrm{~Hz}, 1 \mathrm{H}), 7.46(\mathrm{t}, J=7.8 \mathrm{~Hz}, 1 \mathrm{H}), 7.37(\mathrm{t}, J=7.5 \mathrm{~Hz}, 2 \mathrm{H}), 7.13(\mathrm{dd}, J=16.4,6.8 \mathrm{~Hz}, 3 \mathrm{H})$, 6.93-6.80 (m, 2H), $6.73(\mathrm{t}, J=7.6 \mathrm{~Hz}, 1 \mathrm{H}), 6.63(\mathrm{~d}, J=8.3 \mathrm{~Hz}, 1 \mathrm{H}), 5.40(\mathrm{~d}, J=12.4 \mathrm{~Hz}, 1 \mathrm{H}), 4.40$ $(\mathrm{d}, J=3.6 \mathrm{~Hz}, 1 \mathrm{H}), 4.09(\mathrm{q}, J=7.1 \mathrm{~Hz}, 1 \mathrm{H}), 3.91(\mathrm{~d}, J=12.2 \mathrm{~Hz}, 1 \mathrm{H}), 3.15(\mathrm{dd}, J=12.3,3.5 \mathrm{~Hz}, 1 \mathrm{H})$, 2.96-2.84 (m, 1H), $2.56(\mathrm{~s}, 2 \mathrm{H}), 2.23(\mathrm{dd}, J=15.2,8.6 \mathrm{~Hz}, 1 \mathrm{H}), 2.17-2.07$ (m, 1H), 1.99 (dd, $J=25.2$, $14.2 \mathrm{~Hz}, 2 \mathrm{H}), 1.70$ (d, $J=6.5 \mathrm{~Hz}, 1 \mathrm{H}) .{ }^{13} \mathrm{C}-\mathrm{NMR}$ (DMSO) $\delta 180.0,174.8,171.9,158.2,143.2,134.5$, $134.1,133.5,133.1,131.8,131.5,130.2,130.1,129.7,128.9,128.4,128.1,127.8,127.4,125.1,122.8$, 119.8, 116.4, 113.1, 112.1, 69.7, 69.6, 63.2, 57.7, 47.5, 30.4, 25.2, 22.6. ESI-MS ( $\mathrm{m} / \mathrm{z})$ : calcd. 668.2, found $668.2\left([\mathrm{M}+\mathrm{H}]^{+}\right)$; HRMS $(\mathrm{m} / z)$ : calcd. $\mathrm{C}_{37} \mathrm{H}_{32} \mathrm{~N}_{5} \mathrm{NiO}_{4}$ for 668.1808 , found $668.1819\left([\mathrm{M}+\mathrm{H}]^{+}\right)$. HPLC (Chiralpak IA, $n$-hexane $i$-propanol $=50 / 50$, flow rate $1.0 \mathrm{~mL} / \mathrm{min}, \lambda=220 \mathrm{~nm}$ ), $\mathrm{t}_{\text {major }}=14.7 \mathrm{~min}$, $\mathrm{t}_{\text {minor }}=7.1 \mathrm{~min}, \mathrm{de}=98 \%$.

3.3.12. Ni(II)-(S)-BPB/(2S,3R)-2-Amino-4,4'-dicyano-3-(2-fluoro-4-bromophenyl) Butyric Acid Schiff Base Complex (7l)

Yield $=90 \%$, m.p. $222-224{ }^{\circ} \mathrm{C} .[\alpha]_{\mathrm{D}}^{18}=+1624$ (ca. $\left.0.2 \mathrm{~g} / 100 \mathrm{~mL}, \mathrm{CHCl}_{3}\right) .{ }^{1} \mathrm{H}-\mathrm{NMR}\left(\mathrm{CDCl}_{3}\right) \delta 8.32$ $(\mathrm{d}, J=8.7 \mathrm{~Hz}, 1 \mathrm{H}), 8.01(\mathrm{~d}, J=7.5 \mathrm{~Hz}, 2 \mathrm{H}), 7.66(\mathrm{~d}, J=6.2 \mathrm{~Hz}, 3 \mathrm{H}), 7.60(\mathrm{~d}, J=8.6 \mathrm{~Hz}, 2 \mathrm{H}), 7.35(\mathrm{~d}$, $J=5.2 \mathrm{~Hz}, 1 \mathrm{H}), 7.29(\mathrm{dd}, J=13.5,6.0 \mathrm{~Hz}, 3 \mathrm{H}), 7.24-7.11(\mathrm{~m}, 3 \mathrm{H}), 6.68(\mathrm{~s}, 2 \mathrm{H}), 5.23(\mathrm{~d}, J=12.0 \mathrm{~Hz}$, $1 \mathrm{H}), 4.53(\mathrm{~d}, J=3.3 \mathrm{~Hz}, 1 \mathrm{H}), 4.20-4.05(\mathrm{~m}, 1 \mathrm{H}), 3.80(\mathrm{~d}, J=9.4 \mathrm{~Hz}, 1 \mathrm{H}), 3.45(\mathrm{~d}, J=12.6 \mathrm{~Hz}, 1 \mathrm{H})$, $3.30(\mathrm{t}, J=8.5 \mathrm{~Hz}, 1 \mathrm{H}), 2.96(\mathrm{q}, J=10.3 \mathrm{~Hz}, 1 \mathrm{H}), 2.44-2.25(\mathrm{~m}, 1 \mathrm{H}), 2.25-2.08(\mathrm{~m}, 1 \mathrm{H}), 2.02-1.89$ $(\mathrm{m}, 2 \mathrm{H}), 1.79-1.64(\mathrm{~m}, 1 \mathrm{H}) .{ }^{13} \mathrm{C}-\mathrm{NMR}\left(\mathrm{CDCl}_{3}\right) \delta 180.2,176.3,173.3,160.9,143.2,133.9,133.4$, 133.3, 133.2, 131.4, 130.8, 130.5, 129.4, 128.9, 128.8, 127.4, 127.0, 125.6, 124.1, 123.2, 120.8, 115.2, 111.6, 111.3, 70.6, 69.8, 64.0, 57.6, 55.4, 48.1, 30.5, 24.7, 22.9. ESI-MS $(\mathrm{m} / \mathrm{z})$ : calcd. 748.1, found $748.1\left([\mathrm{M}+\mathrm{H}]^{+}\right)$; HRMS $(\mathrm{m} / \mathrm{z})$ : calcd. $\mathrm{C}_{37} \mathrm{H}_{30} \mathrm{BrFN}_{5} \mathrm{NiO}_{3}$ for 748.0869 , found $748.0881\left([\mathrm{M}+\mathrm{H}]^{+}\right)$. HPLC (Chiralpak IA, $n$-hexane $/ i$-propanol $=50 / 50$, flow rate $1.0 \mathrm{~mL} / \mathrm{min}, \lambda=220 \mathrm{~nm}$ ), $\mathrm{t}_{\text {major }}=36.2 \mathrm{~min}$, $\mathrm{t}_{\text {minor }}=15.3 \mathrm{~min}, \mathrm{de}>99 \%$.

3.3.13. Ni(II)-(S)-BPB/(2S,3R,4S)-2-Amino-4-cyano-5-ethoxy-5-oxo-3-phenylpentanoic Acid Schiff Base Complex (7m)

Yield $=78 \%$, m.p. $192.2-193.5^{\circ} \mathrm{C} .[\alpha]_{\mathrm{D}}^{20}=+2323\left(\right.$ ca. $\left.0.03 \mathrm{~g} / 100 \mathrm{~mL}, \mathrm{CH}_{2} \mathrm{Cl}_{2}\right) .{ }^{1} \mathrm{H}-\mathrm{NMR}\left(\mathrm{CDCl}_{3}\right)$ $\delta 8.24(\mathrm{~d}, J=8.6 \mathrm{~Hz}, 1 \mathrm{H}), 7.99(\mathrm{~d}, J=7.1 \mathrm{~Hz}, 2 \mathrm{H}), 7.74-7.58(\mathrm{~m}, 3 \mathrm{H}), 7.53(\mathrm{~s}, 3 \mathrm{H}), 7.40(\mathrm{~d}, J=7.3 \mathrm{~Hz}$, 1H), 7.35-7.26 (m, 4H), 7.22-7.08 (m, 3H), 6.70 (q, $J=7.7 \mathrm{~Hz}, 2 \mathrm{H}), 4.63(\mathrm{~s}, 1 \mathrm{H}), 4.57$ (d, $J=12.0 \mathrm{~Hz}$, $1 \mathrm{H}), 4.18(\mathrm{~d}, J=12.6 \mathrm{~Hz}, 1 \mathrm{H}), 3.85(\mathrm{q}, J=6.9 \mathrm{~Hz}, 2 \mathrm{H}), 3.39(\mathrm{t}, J=12.9 \mathrm{~Hz}, 2 \mathrm{H}), 3.22(\mathrm{t}, J=8.4 \mathrm{~Hz}$, $1 \mathrm{H}), 2.93(\mathrm{dt}, J=9.3,4.6 \mathrm{~Hz}, 1 \mathrm{H}), 2.17(\mathrm{dt}, J=16.0,8.1 \mathrm{~Hz}, 1 \mathrm{H}), 2.02(\mathrm{dd}, J=12.6,6.5 \mathrm{~Hz}, 1 \mathrm{H})$, $1.94(\mathrm{dd}, J=18.3,8.6 \mathrm{~Hz}, 1 \mathrm{H}), 1.82(\mathrm{dt}, J=19.5,6.8 \mathrm{~Hz}, 1 \mathrm{H}), 1.47$ (ddd, $J=19.2,12.4,6.7 \mathrm{~Hz}, 1 \mathrm{H})$, $0.90(\mathrm{t}, J=6.9 \mathrm{~Hz}, 3 \mathrm{H}) .{ }^{13} \mathrm{C}-\mathrm{NMR}\left(\mathrm{CDCl}_{3}\right) \delta 180.28,176.58,173.02,164.34,143.18,134.17,133.89$, $133.71,133.30,132.85,131.46,130.59,130.36,129.32,129.19,129.12,128.88,128.80,127.70$, $127.12,125.83,123.20,120.67,114.69,71.09,70.50,63.81,62.42,57.36,48.45,38.92,30.62,23.06$, 
13.48. HRMS $(\mathrm{m} / z)$ : calcd. $\mathrm{C}_{39} \mathrm{H}_{36} \mathrm{~N}_{4} \mathrm{NaNiO}_{5}{ }^{+}$for 721.1931 , found $721.1931\left([\mathrm{M}+\mathrm{Na}]^{+}\right)$. HPLC (Chiralpak IA, $n$-hexane $/ i$-propanol $=50 / 50$, flow rate $1.0 \mathrm{~mL} / \mathrm{min}, \lambda=220 \mathrm{~nm}$ ), $\mathrm{t}_{\text {major }}=31.7 \mathrm{~min}$, $\mathrm{t}_{\text {minor }}=8.0 \mathrm{~min}, \mathrm{de}=98 \%$.

3.3.14. Ni(II)-(S)-BPB/(2S,3R,4S)-2-Amino-3-(2-bromophenyl)-4-cyano-5-ethoxy-5-oxopentanoic Acid Schiff Base Complex (7n)

Yield $=88 \%$, m.p. $191.3-192.1^{\circ} \mathrm{C} .[\alpha]_{\mathrm{D}}^{20}=+2120\left(\right.$ ca. $\left.0.03 \mathrm{~g} / 100 \mathrm{~mL}, \mathrm{CH}_{2} \mathrm{Cl}_{2}\right) .{ }^{1} \mathrm{H}-\mathrm{NMR}\left(\mathrm{CDCl}_{3}\right)$ $\delta 8.44(\mathrm{~d}, J=8.7 \mathrm{~Hz}, 1 \mathrm{H}), 7.96(\mathrm{~d}, J=7.3 \mathrm{~Hz}, 2 \mathrm{H}), 7.88(\mathrm{~d}, J=7.8 \mathrm{~Hz}, 1 \mathrm{H}), 7.69-7.61(\mathrm{~m}, 3 \mathrm{H})$, $7.58-7.53(\mathrm{~m}, 1 \mathrm{H}), 7.53-7.44(\mathrm{~m}, 2 \mathrm{H}), 7.43-7.36(\mathrm{~m}, 1 \mathrm{H}), 7.29(\mathrm{dd}, J=10.5,4.9 \mathrm{~Hz}, 3 \mathrm{H}), 7.16$ (dt, $J=13.7,5.3 \mathrm{~Hz}, 2 \mathrm{H}), 6.73-6.63(\mathrm{~m}, 2 \mathrm{H}), 4.62(\mathrm{~d}, J=3.0 \mathrm{~Hz}, 1 \mathrm{H}), 4.49(\mathrm{~d}, J=12.2 \mathrm{~Hz}, 1 \mathrm{H}), 4.17(\mathrm{~d}$, $J=12.6 \mathrm{~Hz}, 1 \mathrm{H}), 4.06(\mathrm{dd}, J=12.2,3.0 \mathrm{~Hz}, 1 \mathrm{H}), 3.89(\mathrm{qd}, J=7.1,2.3 \mathrm{~Hz}, 2 \mathrm{H}), 3.43(\mathrm{~d}, J=12.6 \mathrm{~Hz}$, $1 \mathrm{H}), 3.25$ (t, $J=8.6 \mathrm{~Hz}, 1 \mathrm{H}), 2.85-2.75(\mathrm{~m}, 1 \mathrm{H}), 2.19$ (ddd, $J=19.3,13.1,7.3 \mathrm{~Hz}, 2 \mathrm{H}), 1.92$ (dt, $J=11.2,8.1 \mathrm{~Hz}, 1 \mathrm{H}), 1.70(\mathrm{dt}, J=13.6,7.5 \mathrm{~Hz}, 1 \mathrm{H}), 1.46(\mathrm{dt}, J=18.8,6.4 \mathrm{~Hz}, 1 \mathrm{H}), 0.95(\mathrm{t}, J=7.1 \mathrm{~Hz}$, $3 \mathrm{H}) .{ }^{13} \mathrm{C}-\mathrm{NMR}\left(\mathrm{CDCl}_{3}\right) \delta 180.14,176.38,174.21,163.71,143.31,134.68,134.06,133.87,133.70$, $133.24,133.05,131.42,130.80,130.31,130.03,129.76,128.87,128.79,128.55,128.06,127.12$, 127.05, 125.84, 122.92, 120.52, 113.84, 71.89, 70.74, 63.75, 62.67, 57.14, 46.61, 39.74, 30.89, 23.02, 13.50, 0.01. HRMS $(\mathrm{m} / \mathrm{z})$ : calcd. $\mathrm{C}_{39} \mathrm{H}_{35} \mathrm{BrN}_{4} \mathrm{NaNiO}_{5}{ }^{+}$for 799.1037, found 799.1034 $\left([\mathrm{M}+\mathrm{Na}]^{+}\right)$. HPLC (Chiralpak IA, $n$-hexane $/ i$-propanol $=50 / 50$, flow rate $1.0 \mathrm{~mL} / \mathrm{min}, \lambda=220 \mathrm{~nm}$ ), $\mathrm{t}_{\text {major }}=42.8 \mathrm{~min}$, $\mathrm{t}_{\text {minor }}=10.6 \mathrm{~min}, \mathrm{de}>99 \%$.

3.3.15. Ni(II)-(S)-BPB/(2S,3R,4S)-2-Amino-(3-chlorophenyl)-4-cyano-5-ethoxy-3-5-oxopentanoic Acid Schiff Base Complex (7o)

Yield $=89 \%$, m.p. $191.2-193.4{ }^{\circ} \mathrm{C} .[\alpha]_{\mathrm{D}}^{20}=+2376$ (ca. $\left.0.03 \mathrm{~g} / 100 \mathrm{~mL}, \mathrm{CH}_{2} \mathrm{Cl}_{2}\right) .{ }^{1} \mathrm{H}-\mathrm{NMR}\left(\mathrm{CDCl}_{3}\right)$ $\delta 8.27(\mathrm{~d}, J=8.7 \mathrm{~Hz}, 1 \mathrm{H}), 8.00(\mathrm{~d}, J=7.4 \mathrm{~Hz}, 2 \mathrm{H}), 7.70(\mathrm{dd}, J=11.3,4.9 \mathrm{~Hz}, 1 \mathrm{H}), 7.67-7.59(\mathrm{~m}, 2 \mathrm{H})$, $7.53(\mathrm{~d}, J=8.2 \mathrm{~Hz}, 1 \mathrm{H}), 7.46(\mathrm{t}, J=7.8 \mathrm{~Hz}, 1 \mathrm{H}), 7.42-7.34(\mathrm{~m}, 2 \mathrm{H}), 7.31(\mathrm{t}, J=7.6 \mathrm{~Hz}, 2 \mathrm{H}), 7.21-7.13$ $(\mathrm{m}, 3 \mathrm{H}), 7.11(\mathrm{~d}, J=7.2 \mathrm{~Hz}, 1 \mathrm{H}), 6.76-6.64(\mathrm{~m}, 2 \mathrm{H}), 4.62(\mathrm{~d}, J=3.7 \mathrm{~Hz}, 1 \mathrm{H}), 4.53(\mathrm{~d}, J=12.2 \mathrm{~Hz}$, $1 \mathrm{H}), 4.19$ (d, $J=12.6 \mathrm{~Hz}, 1 \mathrm{H}), 3.89$ (q, $J=7.1 \mathrm{~Hz}, 2 \mathrm{H}), 3.42$ (d, $J=12.6 \mathrm{~Hz}, 1 \mathrm{H}), 3.33$ (dd, $J=12.2$, $3.7 \mathrm{~Hz}, 1 \mathrm{H}), 3.26(\mathrm{dd}, J=9.3,7.7 \mathrm{~Hz}, 1 \mathrm{H}), 3.02(\mathrm{dd}, J=10.6,5.8 \mathrm{~Hz}, 1 \mathrm{H}), 2.23(\mathrm{td}, J=17.0,7.6 \mathrm{~Hz}$, $1 \mathrm{H}), 2.09$ (dd, $J=13.4,7.4 \mathrm{~Hz}, 1 \mathrm{H}), 1.99$ (dd, $J=10.8,6.3 \mathrm{~Hz}, 1 \mathrm{H}), 1.93$ (dd, $J=14.0,7.2 \mathrm{~Hz}, 1 \mathrm{H}$ ), $1.62(\mathrm{~d}, J=12.7 \mathrm{~Hz}, 1 \mathrm{H}), 0.96(\mathrm{t}, J=7.1 \mathrm{~Hz}, 3 \mathrm{H}) .{ }^{13} \mathrm{C}-\mathrm{NMR}() \delta 180.29,176.27,173.26,164.17$, $143.30,136.44,135.58,133.91,133.62,133.34,133.04,131.45,130.68,130.43,129.42,129.26$, $128.92,128.83,127.58,127.06,125.66,123.25,120.68,114.35,70.79,70.54,63.88,62.61,57.57$, 48.07, 38.81, 30.76, 23.03, 13.51. HRMS (m/z): calcd. $\mathrm{C}_{39} \mathrm{H}_{35} \mathrm{ClN}_{4} \mathrm{NaNiO}_{5}{ }^{+}$for 755.1542, found $755.1541\left([\mathrm{M}+\mathrm{Na}]^{+}\right)$. HPLC (Chiralpak IA, $n$-hexane $/ i$-propanol $=50 / 50$, flow rate $1.0 \mathrm{~mL} / \mathrm{min}$, $\lambda=220 \mathrm{~nm}), \mathrm{t}_{\text {major }}=26.6 \mathrm{~min}, \mathrm{t}_{\text {minor }}=8.1 \mathrm{~min}, d e=97 \%$.

3.3.16. Ni(II)-(S)-BPB/(2S,3R,4S)-2-Amino-4-cyano-5-ethoxy-3-(4-fluorophenyl)-5-oxopentanoic Acid Schiff Base Complex (7p)

Yield $=75 \%$, m.p. $192.3-193.5^{\circ} \mathrm{C} .[\alpha]_{\mathrm{D}}^{20}=+2250$ (ca. $\left.0.03 \mathrm{~g} / 100 \mathrm{~mL}, \mathrm{CH}_{2} \mathrm{Cl}_{2}\right) .{ }^{1} \mathrm{H}-\mathrm{NMR}\left(\mathrm{CDCl}_{3}\right)$ $\delta 8.25(\mathrm{~d}, J=8.7 \mathrm{~Hz}, 1 \mathrm{H}), 8.00(\mathrm{~d}, J=7.5 \mathrm{~Hz}, 2 \mathrm{H}), 7.69(\mathrm{t}, J=7.0 \mathrm{~Hz}, 1 \mathrm{H}), 7.67-7.59(\mathrm{~m}, 2 \mathrm{H})$, 
$7.39(\mathrm{~d}, J=7.3 \mathrm{~Hz}, 1 \mathrm{H}), 7.30(\mathrm{dd}, J=14.3,6.6 \mathrm{~Hz}, 4 \mathrm{H}), 7.22(\mathrm{~d}, J=8.5,2 \mathrm{H}), 7.16(\mathrm{t}, J=7.3 \mathrm{~Hz}, 3 \mathrm{H})$, $6.70(\mathrm{q}, J=8.2 \mathrm{~Hz}, 2 \mathrm{H}), 4.62(\mathrm{~d}, J=3.4 \mathrm{~Hz}, 1 \mathrm{H}), 4.52(\mathrm{~d}, J=12.2 \mathrm{~Hz}, 1 \mathrm{H}), 4.19(\mathrm{~d}, J=12.6 \mathrm{~Hz}, 1 \mathrm{H})$, $3.88(\mathrm{q}, J=7.1 \mathrm{~Hz}, 2 \mathrm{H}), 3.41(\mathrm{~d}, J=12.6 \mathrm{~Hz}, 1 \mathrm{H}), 3.36(\mathrm{dd}, J=12.2,3.5 \mathrm{~Hz}, 1 \mathrm{H}), 3.29-3.22(\mathrm{~m}, 1 \mathrm{H})$, $2.99(\mathrm{dd}, J=10.1,5.7 \mathrm{~Hz}, 1 \mathrm{H}), 2.25(\mathrm{td}, J=16.7,7.6 \mathrm{~Hz}, 1 \mathrm{H}), 2.09$ (dt, $J=16.1,8.6 \mathrm{~Hz}, 1 \mathrm{H}), 1.96$ $(\mathrm{dt}, J=14.1,9.6 \mathrm{~Hz}, 2 \mathrm{H}), 1.66-1.55(\mathrm{~m}, 1 \mathrm{H}), 0.95(\mathrm{t}, J=7.1 \mathrm{~Hz}, 3 \mathrm{H}) .{ }^{13} \mathrm{C}-\mathrm{NMR}\left(\mathrm{CDCl}_{3}\right) \delta 180.32$, $176.43,173.22,164.62,164.23,162.15,143.22,133.90,133.68,133.33,132.97,131.43,130.65$, $130.44,130.04,130.00,129.23,128.93,128.83,127.58,127.09,125.70,123.22,120.70,116.40$, $116.19,114.44,99.99,70.99,70.48,63.91,62.55,57.48,47.82,38.99,30.68,22.94,13.55$. HRMS $(m / z)$ : calcd. $\mathrm{C}_{39} \mathrm{H}_{35} \mathrm{FN}_{4} \mathrm{NaNiO}_{5}{ }^{+}$for 739.1837 , found 739.1837 ([M+Na] $]^{+}$. HPLC (Chiralpak IA, $n$-hexane $/ i$-propanol $=50 / 50$, flow rate $1.0 \mathrm{~mL} / \mathrm{min}, \lambda=220 \mathrm{~nm}$ ), $\mathrm{t}_{\text {major }}=53.2 \mathrm{~min}, \mathrm{t}_{\text {minor }}=6.9 \mathrm{~min}$, $d e=96 \%$.

3.3.17. Ni(II)-(S)-BPB/(2S,3R,4S)-2-Amino-4-cyano-3-(3,4-dichlorophenyl)-5-ethoxy-5-oxopentanoic Acid Schiff Base Complex (7q)

Yield $=82 \%$, m.p. $192.7-194.7^{\circ} \mathrm{C} .[\alpha]_{\mathrm{D}}^{20}=+2353$ (ca. $\left.0.03 \mathrm{~g} / 100 \mathrm{~mL}, \mathrm{CH}_{2} \mathrm{Cl}_{2}\right) .{ }^{1} \mathrm{H}-\mathrm{NMR}\left(\mathrm{CDCl}_{3}\right) \delta$ $8.27(\mathrm{~d}, J=8.7 \mathrm{~Hz}, 1 \mathrm{H}), 8.02(\mathrm{~d}, J=7.6 \mathrm{~Hz}, 2 \mathrm{H}), 7.70(\mathrm{t}, J=7.1 \mathrm{~Hz}, 1 \mathrm{H}), 7.63(\mathrm{dd}, J=13.5,8.8 \mathrm{~Hz}$, $3 \mathrm{H}), 7.44(\mathrm{~s}, 1 \mathrm{H}), 7.38(\mathrm{~d}, J=7.4 \mathrm{~Hz}, 1 \mathrm{H}), 7.31(\mathrm{t}, J=7.6 \mathrm{~Hz}, 2 \mathrm{H}), 7.20-7.11(\mathrm{~m}, 3 \mathrm{H}), 7.08(\mathrm{~d}, J=7.6 \mathrm{~Hz}$, $1 \mathrm{H}), 6.78-6.64(\mathrm{~m}, 2 \mathrm{H}), 4.60(\mathrm{~d}, J=3.6 \mathrm{~Hz}, 1 \mathrm{H}), 4.51(\mathrm{~d}, J=12.1 \mathrm{~Hz}, 1 \mathrm{H}), 4.19$ (d, $J=12.5 \mathrm{~Hz}, 1 \mathrm{H})$, $3.94(\mathrm{q}, J=7.1 \mathrm{~Hz}, 2 \mathrm{H}), 3.41(\mathrm{~d}, J=12.6 \mathrm{~Hz}, 1 \mathrm{H}), 3.35-3.25(\mathrm{~m}, 2 \mathrm{H}), 3.00(\mathrm{dt}, J=10.2,5.2 \mathrm{~Hz}, 1 \mathrm{H})$, $2.30(\mathrm{dt}, J=16.8,7.7 \mathrm{~Hz}, 1 \mathrm{H}), 2.09(\mathrm{dd}, J=13.3,6.0 \mathrm{~Hz}, 1 \mathrm{H}), 2.02$ (dd, $J=11.8,7.4 \mathrm{~Hz}, 1 \mathrm{H})$, $1.95(\mathrm{dd}, J=13.4,6.3 \mathrm{~Hz}, 1 \mathrm{H}), 1.67(\mathrm{dt}, J=13.0,6.4 \mathrm{~Hz}, 1 \mathrm{H}), 1.02(\mathrm{t}, J=7.1 \mathrm{~Hz}, 3 \mathrm{H}) .{ }^{13} \mathrm{C}-\mathrm{NMR}$ $\left(\mathrm{CDCl}_{3}\right) \delta 180.36,176.15,173.45,164.02,143.31,134.66,133.94,133.80,133.56,133.37,133.17$, $131.42,131.08,130.75,130.50,129.30,128.96,128.86,127.48,127.04,125.53,123.27,120.75$, 114.12, 70.67, 70.54, 64.03, 62.82, 57.78, 47.57, 38.59, 30.75, 22.83, 13.59. HRMS (m/z): calcd. $\mathrm{C}_{39} \mathrm{H}_{34} \mathrm{Cl}_{2} \mathrm{~N}_{4} \mathrm{NaNiO}_{5}{ }^{+}$for 789.1152 , found $789.1151\left([\mathrm{M}+\mathrm{Na}]^{+}\right)$. HPLC (Chiralpak IA, $n$-hexane/ $i$-propanol $=50 / 50$, flow rate $1.0 \mathrm{~mL} / \mathrm{min}, \lambda=220 \mathrm{~nm}), \mathrm{t}_{\text {major }}=44.5 \mathrm{~min}, \mathrm{t}_{\text {minor }}=7.6 \mathrm{~min}, d e=98 \%$.

3.3.18. Ni(II)-(S)-BPB/(2S,3R,4S)-2-Amino-4-cyano-3-(2,4-dichlorophenyl)-5-ethoxy-5-oxopentanoic Acid Schiff Base Complex (7r)

Yield $=96 \%$, m.p. $192.6-194.5^{\circ} \mathrm{C} .[\alpha]_{\mathrm{D}}^{20}=+1960\left(\right.$ ca. $\left.0.03 \mathrm{~g} / 100 \mathrm{~mL}, \mathrm{CH}_{2} \mathrm{Cl}_{2}\right) .{ }^{1} \mathrm{H}-\mathrm{NMR}\left(\mathrm{CDCl}_{3}\right) \delta$ $8.42(\mathrm{~d}, J=8.8 \mathrm{~Hz}, 1 \mathrm{H}), 7.99(\mathrm{~d}, J=7.6 \mathrm{~Hz}, 2 \mathrm{H}), 7.71(\mathrm{~s}, 1 \mathrm{H}), 7.69-7.59(\mathrm{~m}, 3 \mathrm{H}), 7.44(\mathrm{~s}, 2 \mathrm{H}), 7.41$ $(\mathrm{d}, J=3.5 \mathrm{~Hz}, 1 \mathrm{H}), 7.30(\mathrm{dd}, J=13.9,6.7 \mathrm{~Hz}, 3 \mathrm{H}), 7.19-7.11(\mathrm{~m}, 2 \mathrm{H}), 6.72-6.63(\mathrm{~m}, 2 \mathrm{H}), 4.60(\mathrm{~d}$, $J=3.1 \mathrm{~Hz}, 1 \mathrm{H}), 4.48(\mathrm{~d}, J=12.2 \mathrm{~Hz}, 1 \mathrm{H}), 4.18(\mathrm{~d}, J=12.6 \mathrm{~Hz}, 1 \mathrm{H}), 4.05(\mathrm{dd}, J=12.2,3.1 \mathrm{~Hz}, 1 \mathrm{H})$, $3.99-3.88(\mathrm{~m}, 2 \mathrm{H}), 3.43(\mathrm{~d}, J=12.6 \mathrm{~Hz}, 1 \mathrm{H}), 3.34-3.22(\mathrm{~m}, 1 \mathrm{H}), 2.90(\mathrm{dt}, J=11.0,5.6 \mathrm{~Hz}, 1 \mathrm{H}), 2.33$ $(\mathrm{dt}, J=16.8,7.7 \mathrm{~Hz}, 1 \mathrm{H}), 2.16(\mathrm{td}, J=13.6,7.7 \mathrm{~Hz}, 1 \mathrm{H}), 2.00(\mathrm{dt}, J=10.9,7.6 \mathrm{~Hz}, 1 \mathrm{H}), 1.88(\mathrm{dt}$, $J=14.2,7.4 \mathrm{~Hz}, 1 \mathrm{H}), 1.69-1.53(\mathrm{~m}, 1 \mathrm{H}), 1.01(\mathrm{t}, J=7.1 \mathrm{~Hz}, 3 \mathrm{H}) .{ }^{13} \mathrm{C}-\mathrm{NMR}\left(\mathrm{CDCl}_{3}\right) \delta 180.23$, $176.30,174.38,163.63,143.32,137.69,135.70,134.05,133.59,133.31,133.17,131.56,131.39$, $130.83,130.43,130.19,130.14,129.65,128.91,128.83,128.28,127.08,125.58,122.92,120.58$, 113.73, 71.39, 70.71, 63.95, 62.85, 57.45, 43.63, 39.21, 30.98, 22.89, 13.56. HRMS (m/z): calcd. $\mathrm{C}_{39} \mathrm{H}_{34} \mathrm{Cl}_{2} \mathrm{~N}_{4} \mathrm{NaNiO}_{5}{ }^{+}$for 789.1152 , found $789.1151\left([\mathrm{M}+\mathrm{Na}]^{+}\right)$. HPLC (Chiralpak IA, $n$-hexane/ $i$-propanol $=50 / 50$, flow rate $1.0 \mathrm{~mL} / \mathrm{min}, \lambda=220 \mathrm{~nm}), \mathrm{t}_{\text {major }}=77.3, \mathrm{t}_{\text {minor }}=8.8 \mathrm{~min}, d e=98 \%$. 
3.3.19. Ni(II)-(S)-BPB/(2S,3R,4S)-2-Amino-4-cyano-5-ethoxy-5-oxo-3-(p-tolyl)pentanoic Acid Schiff Base Complex (7s)

Yield $=76 \%$, m.p. $182.3-183.7^{\circ} \mathrm{C} .[\alpha]_{\mathrm{D}}^{20}=+2570$ (ca. $\left.0.03 \mathrm{~g} / 100 \mathrm{~mL}, \mathrm{CH}_{2} \mathrm{Cl}_{2}\right) .{ }^{1} \mathrm{H}-\mathrm{NMR}\left(\mathrm{CDCl}_{3}\right)$ $\delta 8.24(\mathrm{~d}, J=8.7 \mathrm{~Hz}, 1 \mathrm{H}), 8.00(\mathrm{~d}, J=7.3 \mathrm{~Hz}, 2 \mathrm{H}), 7.68(\mathrm{dt}, J=9.7,4.3 \mathrm{~Hz}, 1 \mathrm{H}), 7.61(\mathrm{dd}, J=9.1$, $5.4 \mathrm{~Hz}, 2 \mathrm{H}), 7.39$ (d, $J=7.4 \mathrm{~Hz}, 1 \mathrm{H}), 7.31(\mathrm{dd}, J=16.1,8.0 \mathrm{~Hz}, 4 \mathrm{H}), 7.21-7.10(\mathrm{~m}, 5 \mathrm{H}), 6.75-6.65$ $(\mathrm{m}, 2 \mathrm{H}), 4.60(\mathrm{~d}, J=3.7 \mathrm{~Hz}, 1 \mathrm{H}), 4.54(\mathrm{~d}, J=12.2 \mathrm{~Hz}, 1 \mathrm{H}), 4.19(\mathrm{~d}, J=12.6 \mathrm{~Hz}, 1 \mathrm{H}), 3.86(\mathrm{q}, J=7.1 \mathrm{~Hz}$, 2H), $3.41(\mathrm{~d}, J=12.6 \mathrm{~Hz}, 1 \mathrm{H}), 3.33(\mathrm{dd}, J=12.2,3.7 \mathrm{~Hz}, 1 \mathrm{H}), 3.26-3.19(\mathrm{~m}, 1 \mathrm{H}), 3.01-2.93(\mathrm{~m}, 1 \mathrm{H})$, $2.43(\mathrm{~s}, 3 \mathrm{H}), 2.20(\mathrm{dt}, J=16.7,7.9 \mathrm{~Hz}, 1 \mathrm{H}), 2.04(\mathrm{dt}, J=13.1,6.8 \mathrm{~Hz}, 1 \mathrm{H}), 1.95(\mathrm{dd}, J=11.1,7.2 \mathrm{~Hz}$, $1 \mathrm{H}), 1.83(\mathrm{tt}, J=15.3,7.6 \mathrm{~Hz}, 1 \mathrm{H}), 1.49(\mathrm{tt}, J=12.8,6.4 \mathrm{~Hz}, 1 \mathrm{H}), 0.94(\mathrm{t}, J=7.1 \mathrm{~Hz}, 3 \mathrm{H}) .{ }^{13} \mathrm{C}-\mathrm{NMR}$ $\left(\mathrm{CDCl}_{3}\right) \delta 180.19,176.62,172.87,164.36,143.12,138.95,133.86,133.71,133.31,132.80,131.45$, $130.97,130.55,130.30,129.97,129.17,128.87,128.79,127.70,127.11,125.83,123.17,120.65$, 114.76, 71.12, 70.55, 63.87, 62.37, 57.40, 48.10, 38.85, 30.47, 22.78, 21.27, 13.51. HRMS (m/z): calcd. $\mathrm{C}_{40} \mathrm{H}_{38} \mathrm{~N}_{4} \mathrm{NaNiO}_{5}{ }^{+}$for 735.2088 , found $735.2089\left([\mathrm{M}+\mathrm{Na}]^{+}\right)$. HPLC (Chiralpak IA, $n$-hexane/ $i$-propanol $=50 / 50$, flow rate $1.0 \mathrm{~mL} / \mathrm{min}, \lambda=220 \mathrm{~nm}), \mathrm{t}_{\text {major }}=46.3 \mathrm{~min}, \mathrm{t}_{\text {minor }}=7.5 \mathrm{~min}, \mathrm{de}>99 \%$.

3.3.20. Ni(II)-(S)-BPB/(2S,3R,4S)-2-Amino-4-cyano-5-ethoxy-3-(4-methoxylphenyl)-5-oxopentanoic Acid Schiff Base Complex (7t)

Yield $=77 \%$, m.p. $188.5-189.4{ }^{\circ} \mathrm{C} .[\alpha]_{\mathrm{D}}^{20}=+2376\left(\right.$ ca. $\left.0.03 \mathrm{~g} / 100 \mathrm{~mL}, \mathrm{CH}_{2} \mathrm{Cl}_{2}\right) .{ }^{1} \mathrm{H}-\mathrm{NMR}\left(\mathrm{CDCl}_{3}\right) \delta$ $8.23(\mathrm{~d}, J=8.7 \mathrm{~Hz}, 1 \mathrm{H}), 8.01(\mathrm{~d}, J=7.5 \mathrm{~Hz}, 2 \mathrm{H}), 7.68(\mathrm{t}, J=6.9 \mathrm{~Hz}, 1 \mathrm{H}), 7.65-7.58(\mathrm{~m}, 2 \mathrm{H}), 7.38(\mathrm{~d}$, $J=7.4 \mathrm{~Hz}, 1 \mathrm{H}), 7.30(\mathrm{t}, J=7.6 \mathrm{~Hz}, 2 \mathrm{H}), 7.21(\mathrm{~d}, J=8.1 \mathrm{~Hz}, 2 \mathrm{H}), 7.15(\mathrm{dd}, J=13.2,6.6 \mathrm{~Hz}, 3 \mathrm{H}), 7.04$ $(\mathrm{d}, J=8.5 \mathrm{~Hz}, 2 \mathrm{H}), 6.74-6.65(\mathrm{~m}, 2 \mathrm{H}), 4.60(\mathrm{~d}, J=3.5 \mathrm{~Hz}, 1 \mathrm{H}), 4.52(\mathrm{~d}, J=12.2 \mathrm{~Hz}, 1 \mathrm{H}), 4.19(\mathrm{~d}$, $J=12.6 \mathrm{~Hz}, 1 \mathrm{H}), 3.91-3.86(\mathrm{~m}, 2 \mathrm{H}), 3.85(\mathrm{~s}, 3 \mathrm{H}), 3.40(\mathrm{~d}, J=12.6 \mathrm{~Hz}, 1 \mathrm{H}), 3.32$ (dd, $J=12.2,3.5$ $\mathrm{Hz}, 1 \mathrm{H}), 3.24(\mathrm{t}, J=8.5 \mathrm{~Hz}, 1 \mathrm{H}), 3.04-2.96(\mathrm{~m}, 1 \mathrm{H}), 2.21(\mathrm{dt}, J=16.3,7.6 \mathrm{~Hz}, 1 \mathrm{H}), 2.08$ (dd, $J=13.3,6.2 \mathrm{~Hz}, 1 \mathrm{H}), 1.98(\mathrm{dd}, J=11.0,6.9 \mathrm{~Hz}, 1 \mathrm{H}), 1.90(\mathrm{dd}, J=13.5,6.8 \mathrm{~Hz}, 1 \mathrm{H}), 1.56-1.47$ (m, $1 \mathrm{H}), 0.95(\mathrm{t}, J=7.1 \mathrm{~Hz}, 3 \mathrm{H}) .{ }^{13} \mathrm{C}-\mathrm{NMR}\left(\mathrm{CDCl}_{3}\right) \delta 180.28,176.63,172.88,164.42,160.34,143.15$, $133.85,133.73,133.37,132.79,131.46,130.56,130.32,129.17,128.88,128.79,127.67,127.13$, $125.85,125.78,123.18,120.65,114.73,114.59,71.20,70.59,63.92,62.37,57.55,55.34,47.89,38.97$, 30.62, 22.94, 13.56. HRMS (m/z): calcd. $\mathrm{C}_{40} \mathrm{H}_{38} \mathrm{~N}_{4} \mathrm{NaNiO}_{6}{ }^{+}$for 751.2037 , found $751.2037\left([\mathrm{M}+\mathrm{Na}]^{+}\right)$. HPLC (Chiralpak IA, $n$-hexane $/ i$-propanol $=50 / 50$, flow rate $1.0 \mathrm{~mL} / \mathrm{min}, \lambda=220 \mathrm{~nm}$ ), $\mathrm{t}_{\text {major }}=71.2 \mathrm{~min}$, $\mathrm{t}_{\text {minor }}=8.5 \mathrm{~min}, d e=97 \%$.

3.3.21. Ni(II)-(S)-BPB/(2S,3R,4S)-2-Amino-4-cyano-5-ethoxy-3-(4-nitrophenyl)-5-oxopentanoic Acid Schiff Base Complex (7u)

Yield $=69 \%$, m.p. $206.5-208.6^{\circ} \mathrm{C} .[\alpha]_{\mathrm{D}}^{20}=+2163$ (ca. $\left.0.03 \mathrm{~g} / 100 \mathrm{~mL}, \mathrm{CH}_{2} \mathrm{Cl}_{2}\right) .{ }^{1} \mathrm{H}-\mathrm{NMR}\left(\mathrm{CDCl}_{3}\right)$ $\delta 8.40(\mathrm{~d}, J=8.5 \mathrm{~Hz}, 2 \mathrm{H}), 8.28(\mathrm{~d}, J=8.7 \mathrm{~Hz}, 1 \mathrm{H}), 7.98(\mathrm{~d}, J=7.5 \mathrm{~Hz}, 2 \mathrm{H}), 7.72(\mathrm{t}, J=7.1 \mathrm{~Hz}, 1 \mathrm{H})$, $7.69-7.60(\mathrm{~m}, 2 \mathrm{H}), 7.48(\mathrm{~d}, J=8.4 \mathrm{~Hz}, 2 \mathrm{H}), 7.41(\mathrm{~d}, J=7.4 \mathrm{~Hz}, 1 \mathrm{H}), 7.30(\mathrm{t}, J=7.6 \mathrm{~Hz}, 2 \mathrm{H})$, $7.21-7.11(\mathrm{~m}, 3 \mathrm{H}), 6.75-6.67(\mathrm{~m}, 2 \mathrm{H}), 4.67(\mathrm{~d}, J=3.5 \mathrm{~Hz}, 1 \mathrm{H}), 4.62(\mathrm{~d}, J=12.1 \mathrm{~Hz}, 1 \mathrm{H}), 4.17(\mathrm{~d}$, $J=12.6 \mathrm{~Hz}, 1 \mathrm{H}), 3.96-3.86(\mathrm{~m}, 2 \mathrm{H}), 3.48(\mathrm{dd}, J=12.1,3.5 \mathrm{~Hz}, 1 \mathrm{H}), 3.40(\mathrm{~d}, J=12.6 \mathrm{~Hz}, 1 \mathrm{H}), 3.23$ $(\mathrm{dd}, J=9.7,7.1 \mathrm{~Hz}, 1 \mathrm{H}), 2.95-2.87(\mathrm{~m}, 1 \mathrm{H}), 2.17(\mathrm{dt}, J=17.7,8.9 \mathrm{~Hz}, 1 \mathrm{H}), 1.97-1.86(\mathrm{~m}, 2 \mathrm{H}), 1.68$ $(\mathrm{dd}, J=17.8,10.7 \mathrm{~Hz}, 1 \mathrm{H}), 1.55-1.46(\mathrm{~m}, 1 \mathrm{H}), 1.00(\mathrm{t}, J=7.1 \mathrm{~Hz}, 3 \mathrm{H}) .{ }^{13} \mathrm{C}-\mathrm{NMR}\left(\mathrm{CDCl}_{3}\right) \delta 180.25$, 
$176.03,173.78,163.86,148.69,143.31,142.00,134.01,133.59,133.32,133.25,131.36,130.83$, 130.64, 129.33, 129.00, 128.89, 127.47, 127.03, 125.46, 124.23, 123.26, 120.85, 113.96, 70.71, 70.32, 63.95, 62.93, 57.36, 47.97, 38.63, 30.64, 22.83, 13.61. HRMS (m/z): calcd. $\mathrm{C}_{39} \mathrm{H}_{35} \mathrm{~N}_{5} \mathrm{NaNiO}_{7}^{+}$for 766.1782, found $766.1782\left([\mathrm{M}+\mathrm{Na}]^{+}\right)$. HPLC (Chiralpak IA, $n$-hexane/ $i$-propanol $=50 / 50$, flow rate $1.0 \mathrm{~mL} / \mathrm{min}, \lambda=220 \mathrm{~nm}), \mathrm{t}_{\text {major }}=69.0 \mathrm{~min}, \mathrm{t}_{\operatorname{minor}}=8.6 \mathrm{~min}, d e=97 \%$.

3.3.22. Ni(II)-(S)-BPB/(2S,3R,4S)-2-Amino-3-(4-(tert-butyl)phenyl)-4-cyano-5-ethoxy-5-oxopentanoic Acid Schiff Base Complex (7v)

Yield $=51 \%$, m.p. $200.8-201.7^{\circ} \mathrm{C} .[\alpha]_{\mathrm{D}}^{20}=+2260\left(\right.$ ca. $\left.0.03 \mathrm{~g} / 100 \mathrm{~mL}, \mathrm{CH}_{2} \mathrm{Cl}_{2}\right) .{ }^{1} \mathrm{H}-\mathrm{NMR}\left(\mathrm{CDCl}_{3}\right)$ $\delta 8.29(\mathrm{~d}, J=8.7 \mathrm{~Hz}, 1 \mathrm{H}), 7.95(\mathrm{~d}, J=7.4 \mathrm{~Hz}, 2 \mathrm{H}), 7.72-7.66(\mathrm{~m}, 1 \mathrm{H}), 7.66-7.59(\mathrm{~m}, 2 \mathrm{H}), 7.50(\mathrm{~d}$, $J=8.3 \mathrm{~Hz}, 2 \mathrm{H}), 7.40(\mathrm{~d}, J=7.4 \mathrm{~Hz}, 1 \mathrm{H}), 7.30(\mathrm{t}, J=7.6 \mathrm{~Hz}, 2 \mathrm{H}), 7.17(\mathrm{dt}, J=15.7,7.6 \mathrm{~Hz}, 5 \mathrm{H})$, 6.75-6.66 (m, 2H), $4.63(\mathrm{~d}, J=3.7 \mathrm{~Hz}, 1 \mathrm{H}), 4.53(\mathrm{~d}, J=12.2 \mathrm{~Hz}, 1 \mathrm{H}), 4.20(\mathrm{~d}, J=12.7 \mathrm{~Hz}, 1 \mathrm{H})$, $3.86-3.76(\mathrm{~m}, 2 \mathrm{H}), 3.49(\mathrm{~d}, J=12.7 \mathrm{~Hz}, 1 \mathrm{H}), 3.32(\mathrm{dd}, J=12.2,3.7 \mathrm{~Hz}, 1 \mathrm{H}), 3.17(\mathrm{dd}, J=9.8,7.4 \mathrm{~Hz}$, $1 \mathrm{H}), 3.00(\mathrm{dd}, J=10.3,6.8 \mathrm{~Hz}, 1 \mathrm{H}), 2.18(\mathrm{dt}, J=18.1,8.3 \mathrm{~Hz}, 1 \mathrm{H}), 2.06(\mathrm{dd}, J=11.2,6.6 \mathrm{~Hz}, 1 \mathrm{H})$, $1.89(\mathrm{dd}, J=19.4,8.6 \mathrm{~Hz}, 1 \mathrm{H}), 1.80(\mathrm{dd}, J=10.3,6.2 \mathrm{~Hz}, 1 \mathrm{H}), 1.48(\mathrm{~d}, J=8.3 \mathrm{~Hz}, 1 \mathrm{H}), 1.34(\mathrm{~s}, 9 \mathrm{H})$, $0.79(\mathrm{t}, J=7.1 \mathrm{~Hz}, 3 \mathrm{H}) .{ }^{13} \mathrm{C}-\mathrm{NMR}\left(\mathrm{CDCl}_{3}\right) \delta 180.08,176.69,173.06,164.52,151.93,143.13,133.93$, $133.77,133.17,132.84,131.41,130.88,130.56,130.34,129.16,128.85,128.80,127.82,127.07$, $126.29,126.25,125.92,123.10,120.69,114.80,70.98,70.48,63.49,62.22,56.56,48.08,39.24,34.76$, 31.35, 30.72, 22.77, 13.35. HRMS $(\mathrm{m} / z)$ : calcd. $\mathrm{C}_{43} \mathrm{H}_{44} \mathrm{~N}_{4} \mathrm{NaNiO}_{5}{ }^{+}$for 777.2557 , found 777.2557 $\left([\mathrm{M}+\mathrm{Na}]^{+}\right)$. HPLC (Chiralpak IA, $n$-hexane $/ i$-propanol $=50 / 50$, flow rate $1.0 \mathrm{~mL} / \mathrm{min}, \lambda=220 \mathrm{~nm}$ ), $\mathrm{t}_{\text {major }}=58.6 \mathrm{~min}, \mathrm{t}_{\text {minor }}=8.7 \mathrm{~min}, d e=97 \%$.

3.3.23. Ni(II)-(S)-BPB/(2S,3R,4S)-2-Amino-4-cyano-5-ethoxy-3-(naphthalen-1-y)-5-oxopentanoic Acid Schiff Base Complex (7w)

Yield $=67 \%$, m.p. $188.4-190.3{ }^{\circ} \mathrm{C} .[\alpha]_{\mathrm{D}}^{20}=+1793$ (ca. $\left.0.03 \mathrm{~g} / 100 \mathrm{~mL}, \mathrm{CH}_{2} \mathrm{Cl}_{2}\right) .{ }^{1} \mathrm{H}-\mathrm{NMR}\left(\mathrm{CDCl}_{3}\right)$ $\delta 8.25(\mathrm{~d}, J=8.7 \mathrm{~Hz}, 1 \mathrm{H}), 8.00(\mathrm{dd}, J=16.3,8.0 \mathrm{~Hz}, 2 \mathrm{H}), 7.90(\mathrm{~d}, J=7.5 \mathrm{~Hz}, 2 \mathrm{H}), 7.71(\mathrm{dd}, J=16.1$, $8.1 \mathrm{~Hz}, 3 \mathrm{H}), 7.66-7.56(\mathrm{~m}, 3 \mathrm{H}), 7.54-7.44(\mathrm{~m}, 2 \mathrm{H}), 7.41(\mathrm{~d}, J=7.7 \mathrm{~Hz}, 1 \mathrm{H}), 7.23(\mathrm{~d}, J=7.4 \mathrm{~Hz}, 2 \mathrm{H})$, $7.18-7.07(\mathrm{~m}, 3 \mathrm{H}), 6.77(\mathrm{~d}, J=8.2 \mathrm{~Hz}, 1 \mathrm{H}), 6.71(\mathrm{t}, J=7.5 \mathrm{~Hz}, 1 \mathrm{H}), 4.80(\mathrm{~s}, 1 \mathrm{H}), 4.76(\mathrm{~d}, J=12.1 \mathrm{~Hz}$, $1 \mathrm{H}), 4.30(\mathrm{~d}, J=10.7 \mathrm{~Hz}, 1 \mathrm{H}), 4.02(\mathrm{~d}, J=12.6 \mathrm{~Hz}, 1 \mathrm{H}), 3.80-3.65(\mathrm{~m}, 2 \mathrm{H}), 3.25(\mathrm{~d}, J=12.5 \mathrm{~Hz}, 1 \mathrm{H})$, $2.94(\mathrm{t}, J=8.7 \mathrm{~Hz}, 1 \mathrm{H}), 2.51(\mathrm{dt}, J=11.5,5.9 \mathrm{~Hz}, 1 \mathrm{H}), 1.89-1.72(\mathrm{~m}, 2 \mathrm{H}), 1.24(\mathrm{dt}, J=13.6,6.8 \mathrm{~Hz}$, 2H), $0.94(\mathrm{dt}, J=21.1,7.7 \mathrm{~Hz}, 1 \mathrm{H}), 0.67(\mathrm{t}, J=7.1 \mathrm{~Hz}, 3 \mathrm{H}) .{ }^{13} \mathrm{C}-\mathrm{NMR}\left(\mathrm{CDCl}_{3}\right) \delta 179.70,176.53$, $173.06,164.07,143.34,134.19,133.94,133.72,133.47,133.23,133.08,131.36,131.22,130.96$, $130.45,129.86,129.42,128.98,128.76,128.69,127.37,126.94,126.86,126.59,126.02,125.80$, $125.42,122.98,122.94,120.40,114.48,72.69,70.32,63.60,62.39,57.23,43.09,39.99,30.34,22.94$, 13.28. HRMS $(\mathrm{m} / \mathrm{z})$ : calcd. $\mathrm{C}_{43} \mathrm{H}_{38} \mathrm{~N}_{4} \mathrm{NaNiO}_{5}{ }^{+}$for 771.2088 , found $771.2088\left([\mathrm{M}+\mathrm{Na}]^{+}\right)$. HPLC (Chiralpak IA, $n$-hexane $/ i$-propanol $=50 / 50$, flow rate $1.0 \mathrm{~mL} / \mathrm{min}, \lambda=220 \mathrm{~nm}$ ), $\mathrm{t}_{\text {major }}=31.7 \mathrm{~min}$, $\mathrm{t}_{\text {minor }}=12.3 \mathrm{~min}, \mathrm{de}>99 \%$. 


\subsection{Procedure for the Synthesis of (2S,3R)-8a}

In a typical procedure, $3 \mathrm{~mol} / \mathrm{L} \mathrm{HCl}(3.33 \mathrm{~mL}, 5.0 \mathrm{mmol})$ was added to a solution of the $(S, 2 S, 3 R)-7 \mathbf{a}$ $(1.0 \mathrm{mmol})$ dissolved in THF $(13 \mathrm{~mL})$. The reaction was stirred for $12 \mathrm{~h}$ or until the red color of the solution disappeared and was then concentrated under vacuum to half of the original volume. In the case of $(S, 2 S, 3 R)-7 \mathbf{a}$, the $(S)$-BPB was recovered from the aqueous portion by extracting with ethyl acetate (EA) and was washed with water. The organic layer was removed, and the aqueous portion was diluted with water $(2 \mathrm{~mL})$. The aqueous portion was transferred to a clean flask, and solid $\mathrm{NaHCO}_{3}$ ( $336 \mathrm{mg}, 4.0 \mathrm{mmol}$ ) was carefully added with stirring to neutralize the solution, followed by $\mathrm{Na}_{2}$ EDTA (372 mg, $1.0 \mathrm{mmol}$ ), and was stirred for $5 \mathrm{~min}$. Additional solid $\mathrm{NaHCO}_{3}$ (336 mg, $4.0 \mathrm{mmol}$ ) was added, followed by a solution of Fmoc-OSu $(337 \mathrm{mg}, 1.0 \mathrm{mmol})$ in acetonitrile $(5 \mathrm{~mL})$. The reaction was stirred for $24 \mathrm{~h}$ under nitrogen, concentrated in vacuum to half of the original volume, adjusted to $\mathrm{pH}=3$ with $10 \%$ citric acid, and extracted with EtOAc twice. Combined organic layers were washed with brine, dried with anhydrous $\mathrm{MgSO}_{4}$, concentrated, and purified on silica gel using a flash chromatography (petroleum ether/ethyl acetate $=1 / 2)$ to give $(2 S, 3 R)-\mathbf{8 a}$ as a white solid.

\subsection{2-(((9H-Fluoren-9-yl)methoxy)carbonyl)-4,4-dicyano-3-phenylbutanoic Acid (8a)}

${ }^{1} \mathrm{H}-\mathrm{NMR}\left(\mathrm{CDCl}_{3}\right) \delta 8.02(\mathrm{~s}, 1 \mathrm{H}), 7.68-7.54(\mathrm{~m}, 3 \mathrm{H}), 7.54-7.41(\mathrm{~m}, 2 \mathrm{H}), 7.21-6.97(\mathrm{~m}, 6 \mathrm{H})$, $6.58(\mathrm{~s}, 1 \mathrm{H}), 4.42(\mathrm{~s}, 1 \mathrm{H}), 4.35-4.17(\mathrm{~m}, 2 \mathrm{H}), 4.11(\mathrm{~s}, 1 \mathrm{H}), 4.06(\mathrm{~s}, 1 \mathrm{H}), 3.82(\mathrm{~s}, 1 \mathrm{H}) .{ }^{13} \mathrm{C}-\mathrm{NMR}$ (DMSO) $\delta 172.8,162.3,150.4,143.2,140.6,140.6,128.9,128.8,127.8,127.7,127.2,127.2$, 127.1, 126.4, 121.3, 120.1, 120.0, 109.7, 67.8, 59.7, 46.1, 25.1, 20.7. ESI-MS $(\mathrm{m} / \mathrm{z})$ : calcd. 450.2, found $450.4\left([\mathrm{M}-\mathrm{H}]^{-}\right)$.

\section{Conclusions}

We have reported the first asymmetric three-component reaction of chiral nickel(II) glycinate, aromatic aldehydes, and an $\alpha$-carbanion of two electron-withdrawing groups (malononitrile or ethyl cyanoacetate) to give a series of novel $\alpha$-amino- $\beta$-substituted $\gamma, \gamma$-disubstituted butyric acid derivatives. We have screened a series of reaction conditions and developed a practical system to promote the asymmetric three component reaction of chiral nicke(II) glycinate. This reaction, which constructed two carbon-carbon bonds and formed two or three chiral centers, provides a convenient synthesis of functionalized chiral Fmoc- $\alpha$-amino- $\beta$-substituted $\gamma, \gamma$-disubstituted butyric acid derivatives. The transformation performed well with electron-deficient, electron-rich, condensed ring and sterically hindered aromatic aldehydes and addorded functionalized products. To our excitement, some of them had amazingly high diastereoselectivities, but the heteroaryl substitutes were not well tolerated. The absolute configurations of the typical products were determined. Further studies will focus on mechanistic aspects, expansion of substrate ranges, and further applications of other chiral nickel(II) complexes in important carbon-carbon bond-forming reactions.

\section{Supplementary Materials}

Supplementary materials can be accessed at: http://www.mdpi.com/1420-3049/19/1/826/s1. 


\section{Acknowledgments}

This research has received financial support from the National Natural Science Foundation of China (No. 81001357, 81273471 and 81303208) and the Open Research Fund of State Key Laboratory Breeding Base of Systematic Research, Development and Utilization of Chinese Medicine.

\section{Conflicts of Interest}

The authors declare no conflict of interest.

\section{References}

1. Or, Y.S.; Ying, L.; Wang, C.; Long, J.; Qui, Y.L. Preparation of Substituted Pyrrolidine Derivatives for Use as Anti-Infective Agents. WO 2009003009, 31 December 2008.

2. Edwards, D.L.; Berens, M.E.; Beaudry, C. Identification of Chemical Useful for Treating e.g., Cancer, Comprising Depositing Cancer Cells on Substrate Using Guided Cell Sedimentation, Treating Cancer Cells with Library of Chemicals, and Measuring Cell Migration Rate. WO 2003102153, 11 December 2003.

3. Jones, D.T.; Harris, A.L. Identification of novel small-molecule inhibitors of hypoxia-inducible factor-1 transactivation and DNA binding. Mol. Cancer Ther. 2006, 5, 2193-2202.

4. Dandia, A.; Jain, A.K.; Laxkar, A.K.; Bhati, D.S. Synthesis and stereochemical investigation of highly functionalized novel dispirobisoxindole derivatives via [3+2] cycloaddition reaction in ionic liquid. Tetrahedron 2013, 69, 2062-2069.

5. Erdbrink, H.; Peuser, I.; Gerling, U.I.M.; Lentz, D.; Koksch, B.; Czekelius, C. Conjugate hydrotrifluoromethylation of $\alpha, \beta$-unsaturated acyl-oxazolidinones: Synthesis of chiral fluorinated amino acids. Org. Biomol. Chem. 2012, 10, 8583-8586.

6. Aouadi, K.; Jeanneau, E.; Msaddek, M.; Praly, J.P. 1,3-Dipolar cycloaddition of a chiral nitrone to (E)-1,4-dichloro-2-butene: A new efficient synthesis of $(2 S, 3 S, 4 R)-4$-hydroxyisoleucine. Tetrahedron Lett. 2012, 53, 1817-1821.

7. Tong, B.M.K.; Chiba, S. Diamine-catalyzed conjugate addition to acrylate derivatives. Org. Lett. 2011, 13, 2948-2951.

8. Dugave, C.; Cluzeau, J.; Menez, A.; Gaudry, M.; Marquet, A. Chemo-enzymic synthesis of protected cyano derivatives of glutamate. Tetrahedron Lett. 1998, 39, 5775-5778.

9. Heimgartner, H. 3-Amino-2H-azirines. Synthons for $\alpha, \alpha$-disubstituted $\alpha$-amino acids in heterocycle and peptide synthesis. Angew. Chem. Int. Ed. 1991, 30, 238-264.

10. Duthaler, R.O. Recent developments in the stereoselective synthesis of $\alpha$-amino acids. Tetrahedron 1994, 50, 1539-1650.

11. Wirth, T. New strategies to alpha-alkylated alpha-amino acids. Angew. Chem. Int. Ed. 1997, 36, $225-227$.

12. Gibson, S.E.; Guillo, N.; Tozer, M.J. Towards control of chi-space: Conformationally constrained analogues of Phe, Tyr, Trp and His. Tetrahedron 1999, 55, 585-615.

13. Cativiela, C.; Diaz-de-Villegas, M.D. Stereoselective synthesis of quaternary alpha-amino acids. Part 1: Acyclic compounds. Tetrahedron Asymmetry 1998, 9, 3517-3599. 
14. Cativiela, C.; Diaz-de-Villegas, M.D. Stereoselective synthesis of quaternary alpha-amino acids. Part 2. Cyclic compounds. Tetrahedron Asymmetry 2000, 11, 645-732.

15. Flores-Conde, M.I.; Reyes, L.; Herrera, R.; Rios, H.; Vazquez, M.A.; Miranda, R.; Tamariz, J.; Delgado, F. Highly regio- and stereoselective diels-alder cycloadditions via two-step and multicomponent reactions promoted by infrared irradiation under solvent-free conditions. Int. J. Mol. Sci. 2012, 13, 2590-2617.

16. Belokon, Y.N. Chiral complexes of $\mathrm{Ni}(\mathrm{II}), \mathrm{Cu}(\mathrm{II})$, and $\mathrm{Cu}(\mathrm{I})$ as reagents, catalysts and receptors for asymmetric-synthesis and chiral recognition of amino-acids. Pure Appl. Chem. 1992, 64, 1917-1924.

17. Belokon, Y.N.; Bespalova, N.B.; Churkina, T.D.; Cisarova, I.; Ezernitskaya, M.G.; Harutyunyan, S.R.; Hrdina, R.; Kagan, H.B.; Kocovsky, P.; Kochetkov, K.A.; et al. Synthesis of alpha-amino acids via asymmetric phase transfer-catalyzed alkylation of achiral nickel(II) complexes of glycine-derived Schiff bases. J. Am. Chem. Soc. 2003, 125, 12860-12871.

18. Belokon, Y.N.; Tararov, V.I.; Maleev, V.I.; Saveleva, T.F.; Ryzhov, M.G. Improved procedures for the synthesis of (S)-2- $N$-( $N$ '-benzylprolyl)amino benzophenone (BPB) and Ni(II) complexes of Schiff's bases derived from BPB and amino acids. Tetrahedron Asymmetry 1998, 9, 4249-4252.

19. Hayashi, T.; Kishi, E.; Soloshonok, V.A.; Uozumi, Y. Erythro-selective aldol-type reaction of $N$-sulfonylaldimines with methyl isocyanoacetate catalyzed by gold(I). Tetrahedron Lett. 1996, 37, 4969-4972.

20. Soloshonok, V.A.; Fokina, N.A.; Rybakova, A.V.; Shishkina, I.P.; Galushko, S.V.; Sorochinsky, A.E.; Kukhar, V.P.; Savchenko, M.V.; Svedas, V.K. Biocatalytic approach to enantiomerically pure beta-amino acids. Tetrahedron Asymmetry 1995, 6, 1601-1610.

21. Soloshonok, V.A.; Soloshonok, I.V.; Kukhar, V.P.; Svedas, V.K. Biomimetic transamination of alpha-alkyl beta-keto carboxylic esters. Chemoenzymatic approach to the stereochemically defined alpha-alkyl beta-fluoroalkyl beta-amino acids. J. Org. Chem. 1998, 63, 1878-1884.

22. Deng, G.H.; Wang, J.; Zhou, Y.; Jiang, H.L.; Liu, H. One-pot, large-scale synthesis of Nickel(II) complexes derived from 2- $N$-(alpha-picolyl)amino benzophenone (PABP) and $\alpha$ - or $\beta$-amino acids. J. Org. Chem. 2007, 72, 8932-8934.

23. Lin, D.Z.; Deng, G.H.; Wang, J.; Ding, X.; Jiang, H.L.; Liu, H. Efficient Synthesis of Symmetrical alpha,alpha-Disubstituted beta-Amino Acids and alpha,alpha-Disubstituted Aldehydes via Dialkylation of Nucleophilic beta-Alanine Equivalent. J. Org. Chem. 2010, 75, 1717-1722.

24. Wang, J.; Lin, D.Z.; Shi, J.M.; Ding, X.; Zhang, L.; Jiang, H.L.; Liu, H. Highly enantio- and diastereoselective mannich reaction of a chiral Nickel(II) glycinate with an alpha-imino ester for asymmetric synthesis of a 3-aminoaspartate. Synthesis 2010, 7, 1205-1208.

25. Wang, J.; Zhou, S.B.; Lin, D.Z.; Ding, X.; Jiang, H.L.; Liu, H. Highly diastereo- and enantioselective synthesis of syn-beta-substituted tryptophans via asymmetric Michael addition of a chiral equivalent of nucleophilic glycine and sulfonylindoles. Chem. Commun. 2011, 47, 8355-8257.

26. Wang, J.; Ji, X.; Shi, J.M.; Sun, H.F.; Jiang, H.L.; Liu, H. Diastereoselective Michael reaction of chiral nickel(II) glycinate with nitroalkenes for asymmetric synthesis of beta-substituted alpha,gamma-diaminobutyric acid derivatives in water. Amino Acids 2012, 42, 1685-1694. 
27. Wang, J.; Liu, H.; Acena, J.L.; Houck, D.; Takeda, R.; Moriwaki, H.; Sato, T.; Soloshonok, V.A. Synthesis of bis- $\alpha, \alpha$-amino acids through diastereoselective bis-alkylations of chiral Ni(II)-complexes of glycine. Org. Biomol. Chem. 2013, 11, 4508-4515.

28. Smith, D.J.; Yap, G.P.A.; Kelley, J.A.; Schneider, J.P. Enhanced stereoselectivity of a Cu(II) complex chiral auxiliary in the synthesis of Fmoc-L- $\gamma$-carboxyglutamic acid. J. Org. Chem. 2011, $76,1513-1520$.

29. Xie, X.; Peng, C.; He, G.; Leng, H.-J.; Wang, B.; Huang, W.; Han, B. Asymmetric synthesis of a structurally and stereochemically complex spirooxindole pyran scaffold through an organocatalytic multicomponent cascade reaction. Chem. Commun. 2012, 48, 10487-10489.

30. He, J.; Ouyang, G.; Yuan, Z.; Tong, R.; Shi, J.; Ouyang, L. A facile synthesis of functionalized dispirooxindole derivatives via a three-component 1,3-dipolar cycloaddition reaction. Molecules 2013, 18, 5142-5154.

31. Hou, X.; Luo, H.; Zhong, H.; Wu, F.; Zhou, M.; Zhang, W.; Han, X.; Yan, G.; Zhang, M.; $\mathrm{Lu}, \mathrm{L}$; et al. Analysis of furo 3,2-c tetrahydroquinoline and pyrano 3,2-c tetrahydroquinoline derivatives as antitumor agents and their metabolites by liquid chromatography/electrospray ionization tandem mass spectrometry. Rapid Commun. Mass Spectr. 2013, 27, 1222-1230.

32. Li, X.; Yang, L.; Peng, C.; Xie, X.; Leng, H.-J.; Wang, B.; Tang, Z.-W.; He, G.; Ouyang, L.; Huang, W.; et al. Organocatalytic tandem Morita-Baylis-Hillman-Michael reaction for asymmetric synthesis of a drug-like oxa-spirocyclic indanone scaffold. Chem. Commun. 2013, 49, 8692-8694.

33. Wu, G.; Ouyang, L.; Liu, J.; Zeng, S.; Huang, W.; Han, B.; Wu, F.; He, G.; Xiang, M. Synthesis of novel spirooxindolo-pyrrolidines, pyrrolizidines, and pyrrolothiazoles via a regioselective three-component $3+2$ cycloaddition and their preliminary antimicrobial evaluation. Mol. Diver. 2013, 17, 271-283.

Sample Availability: Samples of the compounds $\mathbf{7 a}-\mathbf{y}$ and $\mathbf{8 a}$ are available from the authors.

(C) 2014 by the authors; licensee MDPI, Basel, Switzerland. This article is an open access article distributed under the terms and conditions of the Creative Commons Attribution license (http://creativecommons.org/licenses/by/3.0/). 